\title{
Composition floristique et état des peuplements ligneux des inselbergs suivant un gradient climatique au Burkina Faso (Afrique de l'Ouest)
}

\author{
Elycée Tindano $^{1}$, Souleymane Ganaba ${ }^{2}$ et Adjima Thiombiano ${ }^{1}$ \\ (1) Université de Ouagadougou, UFR/SVT, Laboratoire de Biologie et Ecologie végétales, BP: 7021 Ouagadougou 03, Burki- \\ na Faso. \\ (2) Institut de l'Environnement et de Recherches Agricoles (INERA), CREAF de Kamboinsée, Département Productions Fo- \\ restières, BP: 7047 Ouagadougou 03, Burkina Faso.
}

\begin{abstract}
Résumé: Au Burkina Faso les études menées sur la végétation des inselbergs sont fragmentaires et insuffisantes. Il existe très peu de données sur la végétation des inselbergs et aucune étude n'a encore été menée sur cette végétation suivant un gradient climatique. L'objectif de cette étude est de décrire la végétation ligneuse des inselbergs des quatre secteurs phytogéographiques du Burkina Faso suivant un gradient climatique nord-sud. Les données ont été collectées dans des placeaux de $30 \mathrm{~m}$ x $30 \mathrm{~m}$, suivant un échantillonnage stratifié et aléatoire. Des indices de diversité, d'équitabilité, de valeur d'importance et de similitude ont été calculés pour la végétation des inselbergs granitiques et gréseux des secteurs phytogéographiques. Un total de 188 relevés a été réalisé. 143 espèces ligneuses ont été recensées et se répartissent inégalement dans les quatre secteurs phytogéographiques. Plus de trois quarts moitié (79\%) de ces espèces se retrouve dans le secteur Sud-soudanien, ce qui le rend plus riche floristiquement. Le secteur le moins riche en espèces est le secteur Nord-sahélien avec 37 espèces $(26 \%)$. La richesse floristique augmente du nord au sud du gradient climatique et met en évidence la variation taxonomique suivant le gradient latitudinal de précipitation. Les 143 espèces ligneuses recensées sur les inselbergs des quatre secteurs phytogéographiques représentent $65 \%$ des espèces ligneuses et $7 \%$ de la flore totale du Burkina Faso. Les facteurs topographie et nature du substrat n'ont pas un effet significatif $(\mathrm{p}>0,05)$ sur la composition floristique des inselbergs. La densité et la surface terrière des peuplements ligneux varient significativement suivant l'altitude $(p<0,05)$. Le climat, particulièrement le niveau de précipitation, reste le principal facteur qui influe sur la répartition des taxa devant la nature du substrat, la topographie et l'anthropisation. Les peuplements ligneux des inselbergs des secteurs sud-sahélien et ord-soudanien sont les moins denses. Ils subissent plus de pression anthropique par rapport aux peuplements ligneux des secteurs Nord-sahélien et Sud-soudanien. Les inselbergs les plus hauts ont une végétation ligneuse plus dense que les inselbergs les plus bas.
\end{abstract}

Mots clés: rochers découverts, flore, climat, conservation.

\section{Species Composition and Ecological Relations of the WoOdy Population of INSELBERgs ALONG a Climatic Gradient in Burkina Faso (West-Africa)}

Summary: In Burkina Faso, studies on inselberg vegetation are sporadic and fragmentary. There is very little data on the vegetation of inselbergs and no study has yet been conducted on this vegetation along a climatic gradient.This study aims at describing the woody vegetation of inselbergs of Burkina Faso's four phytogeographical sectors according to a climatic gradient from north to south. Data were collected in small squares of $900 \mathrm{~m}^{2}(30 \mathrm{~m} \times 30 \mathrm{~m})$ by using a stratified and random sampling. Diversity, equitability, importance value and similarity indices were calculated for the vegetation of granite and sandstone inselbergs of each phytogeographical sector. 188 relevés were performed and 143 woody species were recorded, most of them unevenly distributed in the four phytogéographical sectors. More than three quarters $(79 \%)$ of these species were found in the south-sudanian sector, the lowest species richness was stated in the north-sahelian sector with 37 woody species $(26 \%)$. Species richness increases from the north to the south and highlights the taxonomic variation along the latitudinal gradient of precipitation. The 143 woody species found represent $65 \%$ of the woody species and $7 \%$ of the total flora of Burkina Faso. Topography and nature of the rock have no significant $(p>0,05)$ effect on the floristic composition of the inselbergs. Density and basal area of woody plants varied significantly with altitude $(\mathrm{p}<0,05)$. Climate, especially precipitation level, remains the main factor that affects the distribution of taxa on the inselbergs. Due to higher human pressure, the woody population of south-sahelian and north-sudanian inselbergs was less dense than that of the two other regions.

Key words: opened rocks, flora, climate, conservation.

\section{ARtenzusammensetzung und öKologische Beziehungen der Gehölzbestände Von INSELbergen ENTLANG EINES KLIMAgRAdIENTEN IN BURKINA FASO (WESTAFRIKA)}

Zusammenfassung: Aus Burkina Faso liegen nur fragmentarische und meist kurzfristige Studien der Vegetation von Inselbergen vor. Entsprechend existieren nur wenige Daten; eine Untersuchung entlang eines Klimageradienten wurde noch nicht durchgeführt. Die vorliegende Arbeit, in deren Rahmen Inselberge in allen vier pflanzengeografischen Regionen Burkina Fasos untersucht wurden, schließt diese Lücke. Die Daten wurden auf 188 mittels einer stratifizierten Zufallsprobe ausgewählten Flächen von $30 \mathrm{~m}$ x $30 \mathrm{~m}$ erhoben. Für jeden geografischen Sektor wurden wurden Diversität, Equitabilität, Gewicht und Ähnlichkeitskoeeffizient berechnet. Dabei wurden 143 Gehölzarten gefunden, was $65 \%$ der Gehölz- und $7 \%$ der Gesamtflora von Burkina Faso entspricht. Die meistenArten waren ungleich entlang des Gradienten, wobei mehr als drei Viertel im südsudanischen Sektor vorkamen, während im nordsahelischen Bereich die geringste Artenzahl ermittelt wurde. Topografie und Material der Felsen zeigten keine Korrelation zur Artenzusammensetzung, während die Dichte und die Basalfläche der Gehölze signifikant mit der Höhe der Inselberge korrelierte $(\mathrm{p}<0,05)$. Das Klima, insbesondere die Höhe der Niederschläge, 
erwiesen sich als hauptverantwortlich für die Zusammensetzung der Flora der Inselberge. Wohl auf Grund des dort höheren anthropogenen Drucks ist die Gehölzvegation der südsahelichen und norsudanischen Inserlberge weniger dicht als die der beiden anderen Regionen.

Schlagworte: offene Felsflur, Flora, Klima, Naturschutz.

\section{INTRODUCTION}

L'éveil collectif sur la menace de la biodiversité reste encore théorique et son érosion se poursuit à une vitesse inestimable (SINSIN \& KAMPMANN 2010). Elle se manifeste par une dégradation du couvert végétal à un rythme jamais observé auparavant (INOUSSA et al. 2013).

Au Burkina Faso, la dégradation du couvert végétal et la régression des populations d'espèces ligneuses sont perceptibles avec un taux de réduction de $0,87 \%$ par an (MEDD 2011). La dégradation galopante du couvert végétal en général, et celui des ligneux en particulier, contraint la population à s'orienter vers les écosystèmes azonaux autre fois marginalisés à la recherche de biens et services. C'est le cas des inselbergs dont l'importance pour les populations locales est démontrée par des études antérieures (MÜLLER 2008, TINDANO et al. 2011). Un inselberg se définit comme étant un affleurement rocheux généralement constitué de granite ou de gneiss mais aussi de grès (WHITE 1986). Il est caractérisé par un isolement par rapport au relief environnant et par son indépendance du réseau hydrologique régional (BARTHLOTT et al. 1993; PorembSKi 2007; KouAssi et al. 2009). Les inselbergs, véritables refuges pour les plantes, ont fait l'objet de peu d'études au Burkina Faso et des difficultés se posent quant à la comparaison de leur composition floristique sur l'étendue du territoire (MüLLER 2008). Les études sur les inselbergs restent de ce fait fragmentaires au Burkina Faso. En effet, les rares études menées sur les inselbergs sont celles de Müller (2008), Tindano et al., (2011) dans la zone sahélienne qui ont révélé l'anthropisation de ces écosystèmes. A ces études s'ajoutent celles de KüPPERs \& WiTTIG (1995), WitTig et al. (2000), OuÉDraogo (2009) au sud-est du Burkina Faso. Malgré les études déjà menées, nous ne disposons pas aujourd'hui des données sur la végétation des inselbergs le long du gradient climatique nord-sud du Burkina Faso. Or, le climat est l'un des plus importants facteurs écologiques qui expliquent la distribution des espèces et la dynamique des peuplements végétaux (Thiombiano et al. 2006). D'où la nécessité de disposer des données sur les inselbergs suivant le gradient climatique pour une gestion durable de leur ressources végétales. L'objectif général de cette étude est de caractériser la végétation ligneuse des inselbergs des quatre secteurs phytogéographiques du Burkina Faso. L'étude s'articule autour des hypothèses suivantes: (1) la composition et la diversité floristiques des inselbergs augmentent suivant le gradient climatique nord-sud, (2) les inselbergs sont des zones refuges pour les espèces ligneuses menacées sur les plaines. Pour vérifier ces hypothèses, l'étude se propose (i) de comparer la composition floristique entre les inselbergs des quatre secteurs phytogéographiques, (ii) d'évaluer l'impact anthropique (feu de brousse, agriculture, coupe du bois, pâturage) sur la végétation ligneuse des inselbergs.

\section{Materiel et Methodes}

\subsection{Zone d'étude}

Le Burkina Faso est situé au cœur de l'Afrique de l'Ouest, entre les latitudes $9^{\circ} 02^{\prime}$ et $15^{\circ} 05^{\prime}$ Nord et les longitudes $02^{\circ} 02^{\prime} \mathrm{E}$ et $05^{\circ} 03^{\prime} \mathrm{E}$ (Thiombiano et al. 2006; Sambaré et al., 2010). Le Burkina Faso a été subdivisé en quatre secteurs phytogéographiques sur la base du climat, de la végétation et de la flore par Fontès \& GuINKo, (1995) (Fig. 6).

\subsection{Choix des inselbergs étudiés}

Les inselbergs sont relativement présents le long de notre transect et présentent diverses formes et tailles. Les différentes formes rencontrées sont: les affleurements granitiques et gréseux de quelques $\mathrm{m}(0,5$ à $1 \mathrm{~m})$ au dessus du sol (Fig. 1 et 2), les inselbergs en forme de dôme et les inselbergs tabulaires qui culminent entre 320 et $472 \mathrm{~m}$ (Fig. 3, 4 et 5 ). Le choix des sites s'est basé sur la concentration des inselbergs dans les secteurs phytogéographiques et les inselbergs ont été choisis en fonction de la représentativité, de l'homogénéité floristique et de la physionomique de leur végétation. En se basant sur ces critères quatre inselbergs ont été retenus pour chaque secteur phytogéographique, soit au total seize (16) inselbergs pour toute l'étude (Fig. 6).

Le Burkina Faso est situé au cœur de l'Afrique de l'Ouest, entre les latitudes $9^{\circ} 02^{\prime}$ et $15^{\circ} 05^{\prime}$ Nord et les longitudes $02^{\circ} 02^{\prime} \mathrm{E}$ et $05^{\circ} 03^{\prime} \mathrm{E}$ (Thiombiano et al. 2006; SAmbaré et al., 2010). Le Burkina Faso a été subdivisé en quatre secteurs phytogéographiques sur la base du climat, de la végétation et de la flore par Fontès \& Guinko, (1995) (Fig. 6).

\subsection{Choix des inselbergs étudiés}

Les inselbergs sont relativement présents le long de notre transect et présentent diverses formes et tailles. Les différentes formes rencontrées sont: les affleurements granitiques et gréseux de quelques $\mathrm{m}(0,5 \mathrm{à} 1 \mathrm{~m})$ au dessus du sol (Fig. 1 et 2), les inselbergs en forme de dôme et les inselbergs tabulaires qui culminent entre 320 et $472 \mathrm{~m}$ (Fig. 3, 4 et 5). Le choix des sites s'est basé sur la concentration des inselbergs dans les secteurs phytogéographiques et les inselbergs ont été choisis en fonction de la représentativité, de l'homogénéité floristique et de la physionomique de leur végétation. En se basant sur ces critères quatre inselbergs ont été retenus pour chaque secteur phytogéographique, soit au total seize (16) inselbergs pour toute l'étude (Fig. 6). 


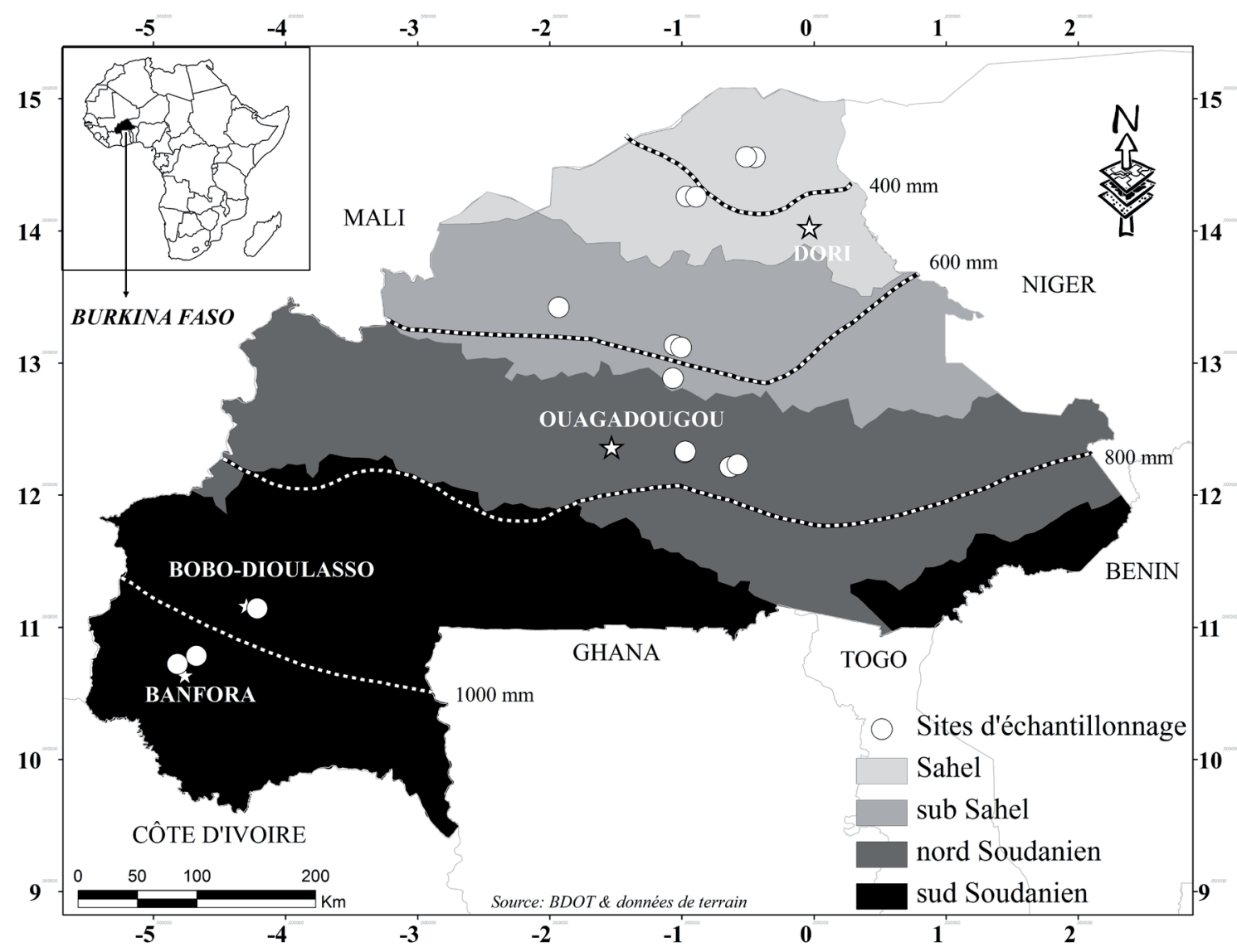

Fig. 1 : Localisation des sites de l'étude / situation of the inselbergs studied.

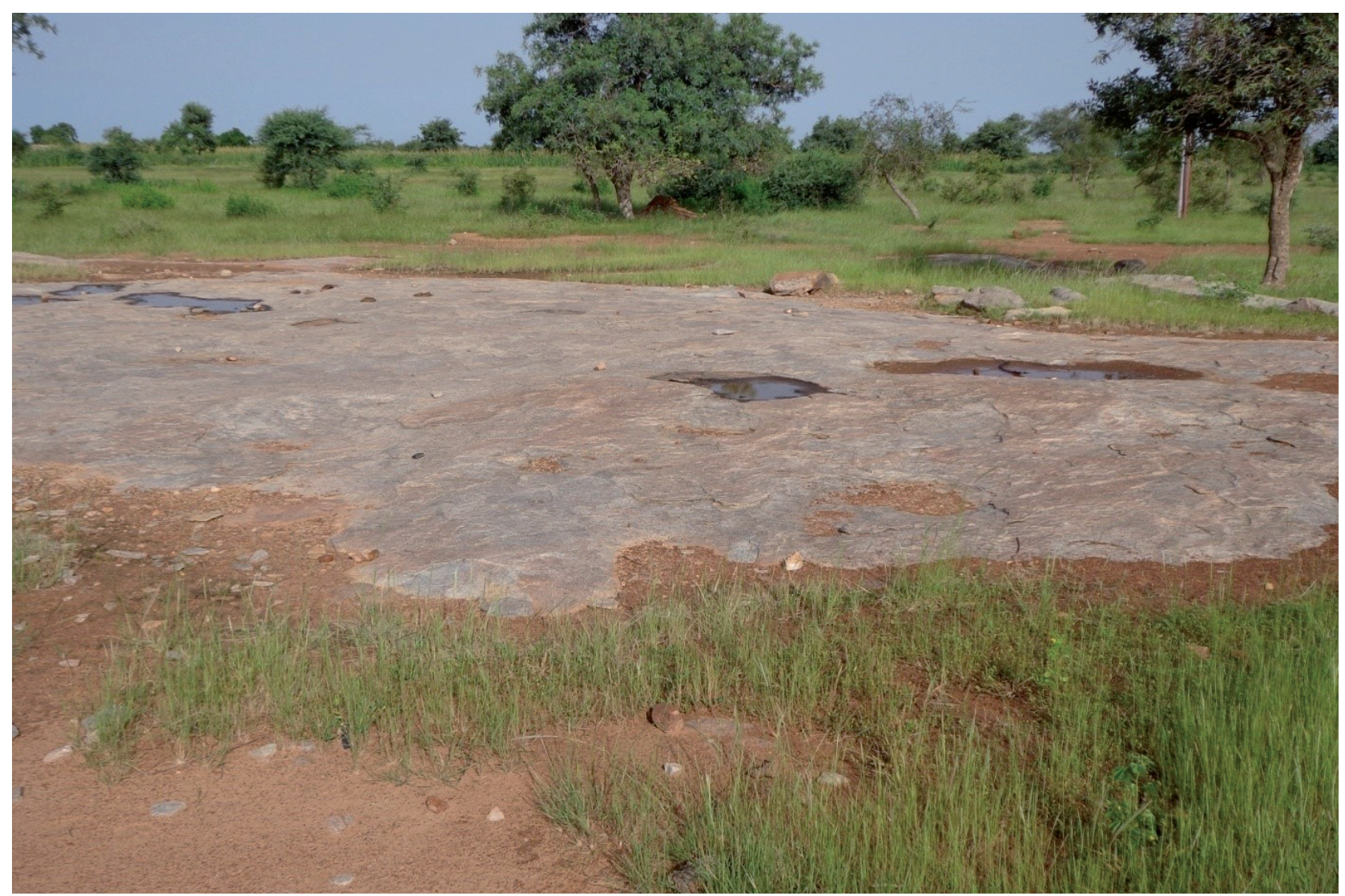

Fig. 2: Affleurement granitique, secteur nord-sahélien, Province de l'Oudalan (Daala) / granite outcrop; North-Sahélien sector, province of Oudalan (Daala); photo E. Tindano. 


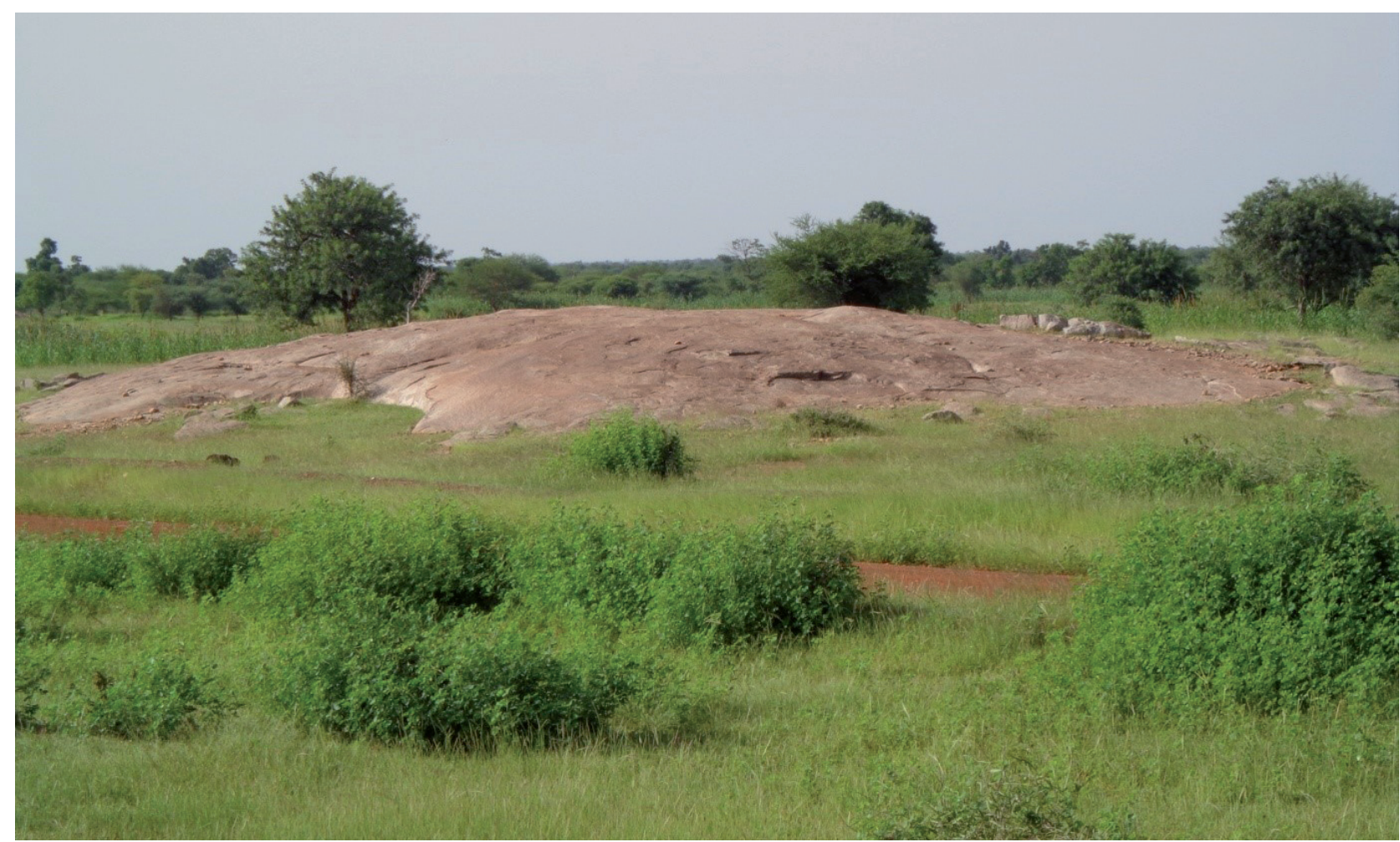

Fig. 3: Affleurement granitique, secteur sud-sahélien, Province du Sanmatenga (Dondolé) / granite outcrop; South-Sahélien sector, province of Sanmatenga (Dondolé); photo E. Tindano.

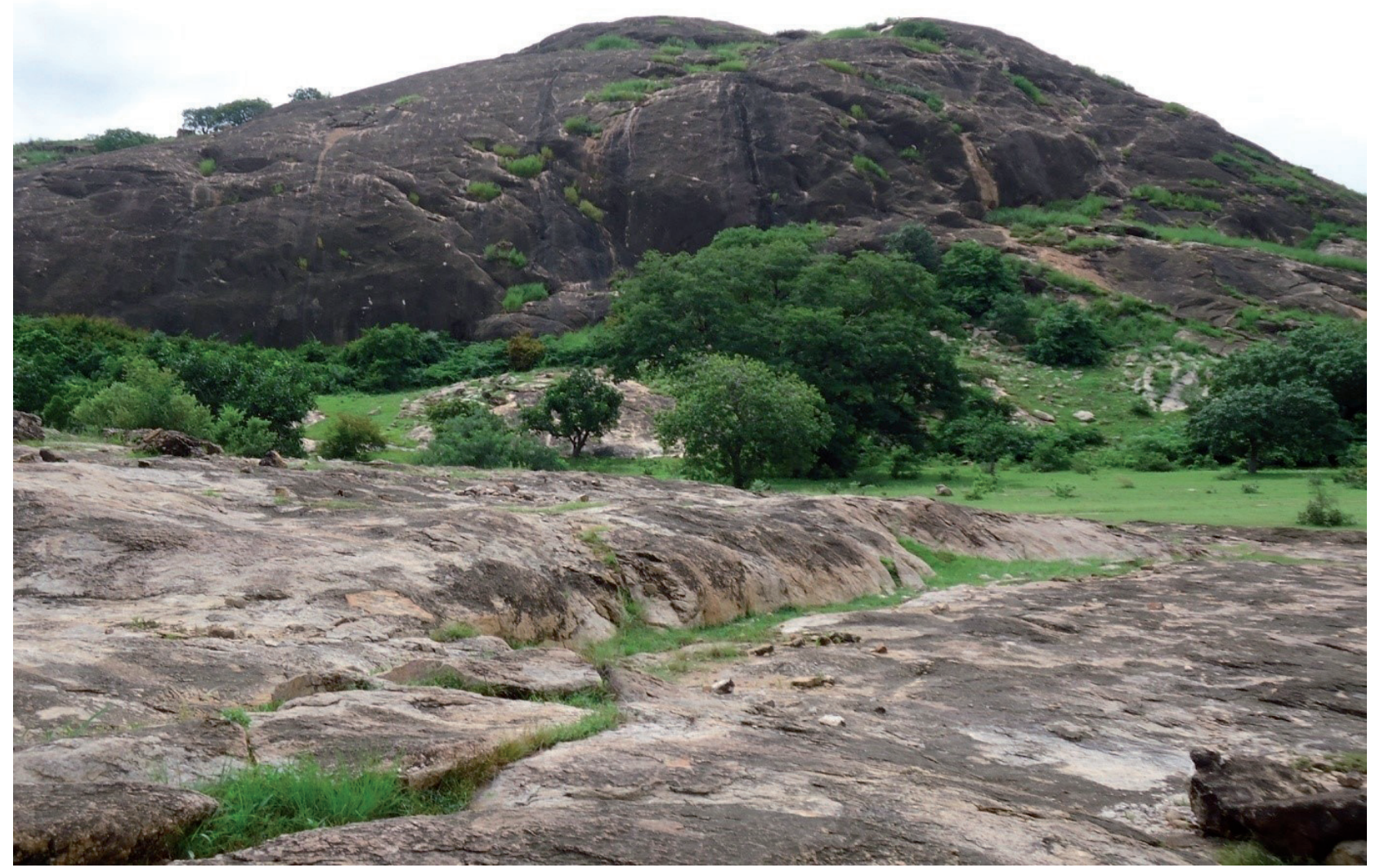

Fig. 4: Inselberg granitique en forme de dôme, secteur nord-soudanien, Province du Ganzourgou (Zorgho) / granite dome-shaped inselberg, NorthSoudanien sector, province of Ganzourgou (Zorgho); photo E. Tindano. 


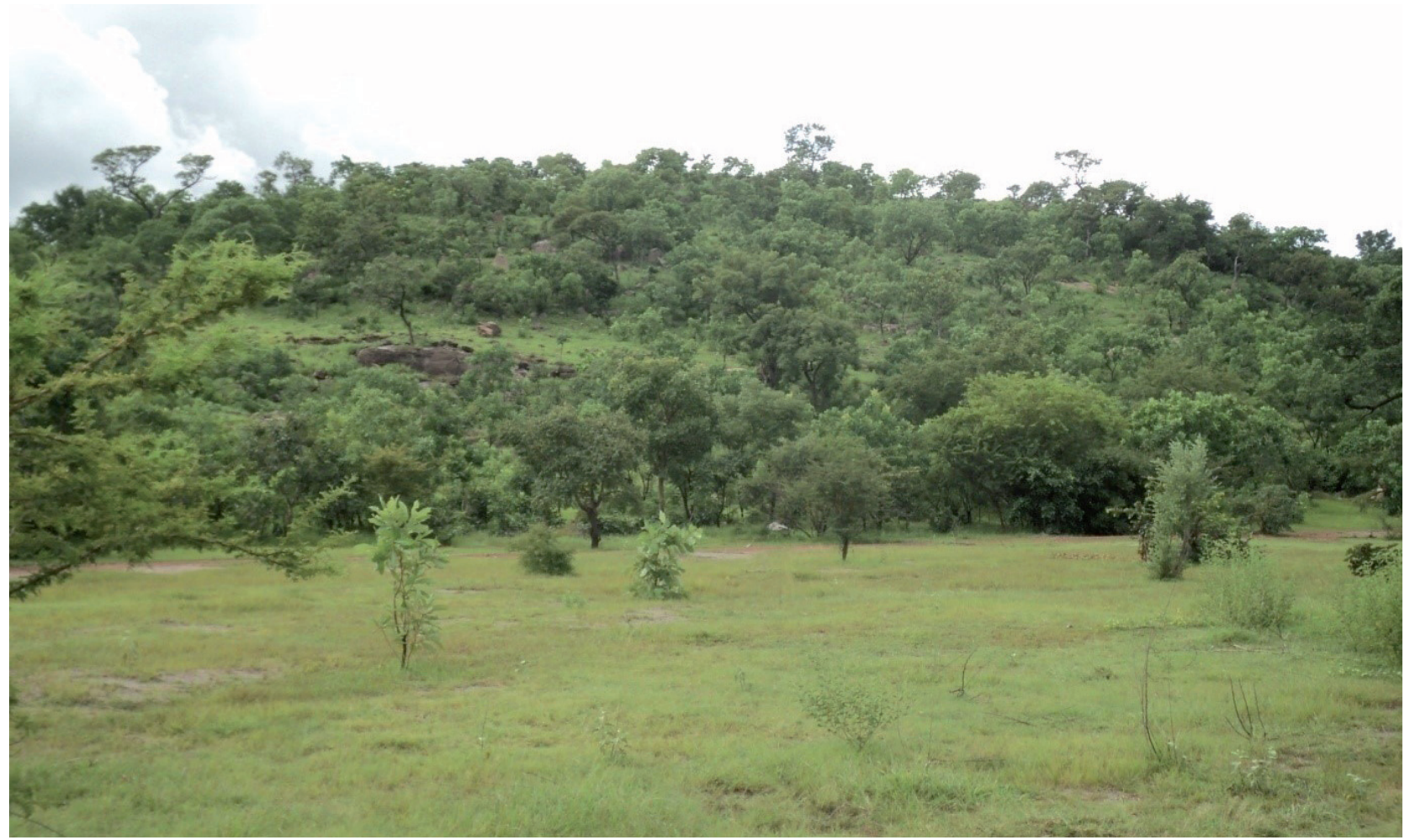

Fig. 5: Inselberg granitique de forme tabulaire, secteur sud-soudanien, Province du Houet (Kotédougou) / granite shield-inselberg from sandstone, South-Soudanien sector, province of Houet (Kotédougou); photo E. Tindano.

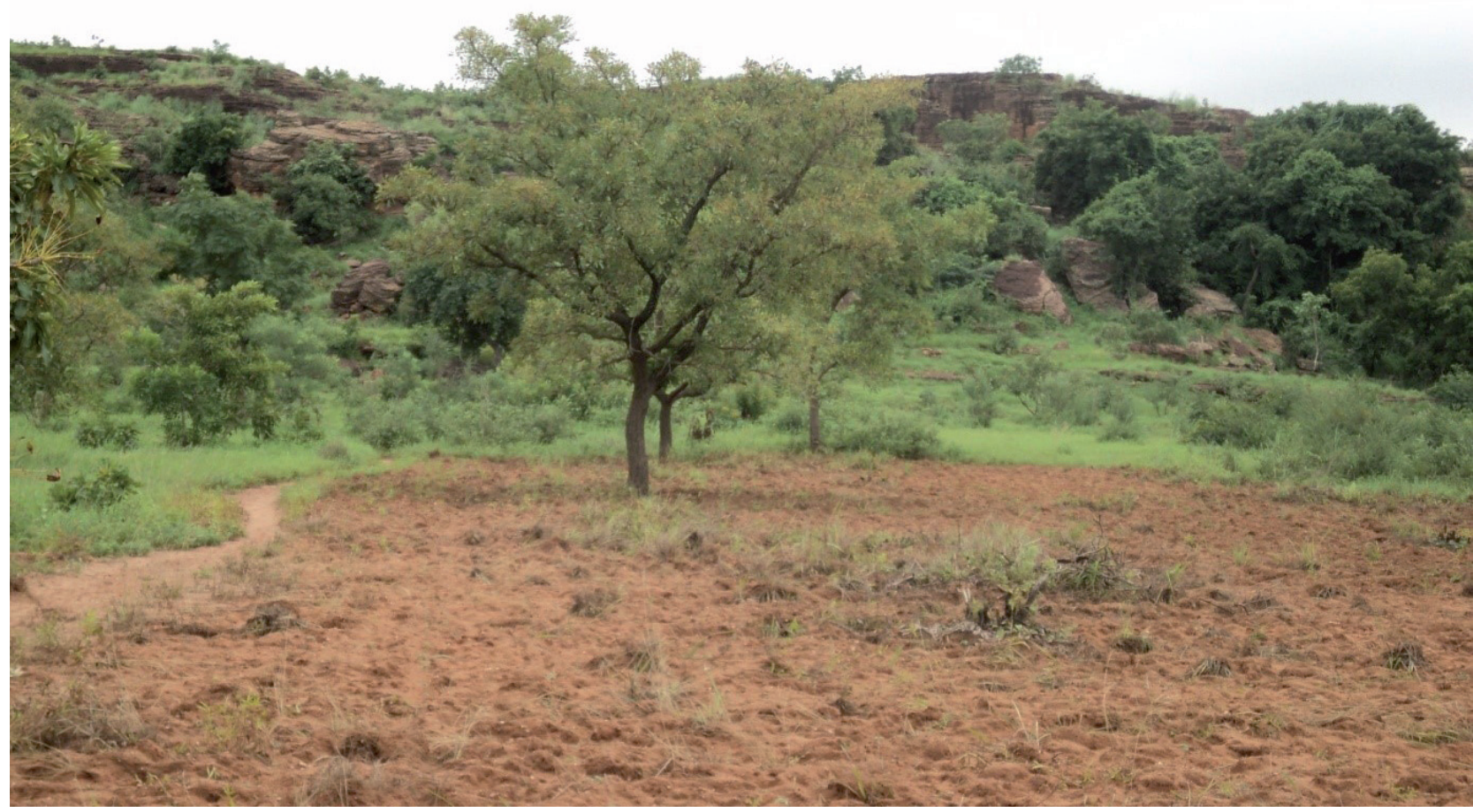

Fig. 6: Inselberg gréseux de forme tabulaire, secteur sud-soudanien, Province du Houet (Kooro) / sandstone-shield-inselberg, South-Soudanien sector, province du Houet (Kooro); photo E. Tindano. 


\subsection{Collecte des données floristiques et dendrométriques}

Pour la caractérisation floristique, des relevés ont été effectués durant les mois d'août 2011 à septembre 2013 sur les versants et les sommets des inselbergs. Les données ont été récoltées suivant un échantillonnage stratifié aléatoire, adopté par TRAORÉ \& MANGARA, (2009). Les placeaux ont été installés sur les versants et les sommets en tenant compte de la nature du substrat (granite, gneiss ou grés) des inselbergs. Ils ont été disposés perpendiculairement au sens de ruissellement de l'eau en tenant compte de l'homogénéité physionomique et floristique de la végétation. La superficie d'un placeau était de $30 \mathrm{~m}$ x $30 \mathrm{~m}\left(900 \mathrm{~m}^{2}\right)$ utilisée par plusieurs auteurs pour l'inventaire de la végétation des inselbergs (KouAssi et al. 2009, PARMENTIER et al. 2006) et bien d'autres écosystèmes (OuÉDRAOGO et al. 2008). Les données phytosociologiques ont été collectées suivant la méthode sigmatiste de Braun-Blanquet (1932). Pour chaque relevé nous avons dressé la liste exhaustive des espèces végétales ligneuses affectées de leur coefficient d'abondance-dominance de Braun-Blanquet. Les données dendrométriques ont été collectées dans les mêmes placettes de $900 \mathrm{~m}^{2}$ que les données phytosociologiques. Les variables collectées sont: le nombre d'individus de chaque espèce ligneuse, le diamètre du tronc des ligneux ayant un diamètre supérieur ou égal à $5 \mathrm{~cm}(\mathrm{~d} \geq 5 \mathrm{~cm}$ ), (OuÉDRAOGO \& THIOMBIANO 2012; TrAORÉ et al. 2012b). Le diamètre a été mesuré à 30 $\mathrm{cm} \mathrm{du}$ sol en raison de la forte ramification de la plus part des espèces de la zone sahélienne (GANABA 2008) et de la déformation des troncs des plantes due à la pression anthropique (BoGNOUNOU 2009).

\subsection{Analyse des données}

\subsubsection{Données floristiques}

Les espèces locales et exotiques inventoriées ont été déterminées à partir des spécimens récoltés et identifiés à l'aide de plusieurs ouvrages (Hutchinson \& Dalziel 1954, 1958, 1963; BÉRHAUT 1967, 1971, 1974, 1975, 1976; АKoEGNinOu et al. 2006; ArbonNier 2009). Les herbiers de 1'Université de Ouagadougou et du Centre National de la Recherche Scientifique et Technologique (CNRST) ont servi également de références pour la comparaison et la détermination des noms des espèces. La nomenclature adoptée dans cet article est celle du catalogue des plantes vasculaire du Burkina Faso (Thiombiano et al. 2012).

Deux catégories de spectres ont été élaborées pour chaque groupe d'inselbergs des secteurs phytogéographiques. Le spectre brut, construit à partir du nombre d'espèces relevant d'un type biologique ou phytogéographique donné et le spectre pondéré est construit à partir de la pondération des espèces par le recouvrement moyen. L'étude ayant portée sur les espèces ligneuses uniquement, les types biologiques concernent les phanérophytes. Ce sont ceux définis par RAUNKIAER (1937) et utilisés dans l'étude de la végétation africaine (MBAYNGONE et al. 2008b; SAMBARÉ et al. 2010; Inoussa et al. 2013) Les types phytogéographiques utilisés proviennent des subdivisions chronologiques de WHITE (1986) utilisés dans plusieurs études antérieures dont celles de OuÉdraogo (2006), Ouoba, 2006, OuÉdraogo et al. (2008).
Pour la comparaison de la diversité floristique des inselbergs les paramètres suivants ont été étudiés: la richesse spécifique moyenne par placeau $(\mathrm{Nm})$, la richesse spécifique totale (Rt), l'indice de diversité de Shannon $(\mathrm{H})$ et l'indice d'équitabilité de Piélou (E). L'indice de similitude de Sørensen (Cs) a été calculé à l'aide de CAP (Communities Analysis Package) version 2. 15, (2002).

- $\mathrm{Cs}=2 \mathrm{C} /(2 \mathrm{~A}+\mathrm{B}+\mathrm{C})$ avec $\mathrm{C}$ : nombre d'espèces communes aux inselbergs; $\mathrm{A}=$ nombre d'espèces des inselbergs du secteur phytogéographique nord-sahélien; $\mathrm{B}=$ nombre d'espèces des inselbergs du secteur phytogéographique sudsoudanien.

- Nm (nombre moyen d'espèces ligneuses recensées par placeau) $=\mathrm{Rt} / \mathrm{Nr}$; $\mathrm{Nr}$ : nombre de relevés

- Rt = nombre total d'espèces ligneuses inventoriées sur les inselbergs de chaque secteur phytogéographique.

$-\mathrm{H}=-\Sigma \mathrm{Pi} \ln \mathrm{Pi}$, Pi (abondance relative des ièmes espèces dans un placeau $)=($ ni $/ \mathrm{N})$, ni: nombre d'individus/espèce, $\mathrm{N}$ : nombre total d'individus par placeau. $\mathrm{H}$ varie en général entre 0 et 5 .

- E=H/lnS, L'équitabilité de Pielou mesure l'abondance relative des différentes espèces constituant la richesse d'une aire. Cela suppose une valeur évoluant entre 0 et 1 avec 1 étant l'équitabilité complète (SAMBARÉ et al. 2011).

- Hmax $=\ln S$, S: nombre total des espèces dans la population.

Des analyses univariées (Anova de Kruskal-Wallis) ont été utilisées pour mesurer la différence de la diversité floristique et des indices de diversités entre les secteurs phytogéographiques.

\subsubsection{Données dendrométriques}

Les paramètres suivants ont été utilisés pour la description de la végétation ligneuse des inselbergs. Il s'agit principalement de:

- la densité $(\mathrm{D}=\mathrm{N} / \mathrm{S}), \mathrm{N}$ : nombre total d'individus ligneux, $\mathrm{S}$ : superficie en hectare, D s'exprime en nombre de pieds/ hectare.

- le diamètre moyen

- la surface terrière $=\mathrm{D}_{20 \mathrm{~cm}}^{2} \pi / 4$ exprimé en $\mathrm{m}^{2} /$ ha

- la dominance relative $=$

Surface terrière totale par espèce

Surface terrière totale de toutes les espèces $\mathrm{x} 100$

- la densité relative $=$

Nombre $d^{\wedge \prime}$ individus par espèce x 100

Nombre total $d^{\wedge \prime}$ individus de toutes les espèces 
- la fréquence $=$

Nombre de relevés où $l^{\wedge \prime}$ espèce est presente $\mathrm{x} 100$

Nombre total des relevés

- la fréquence relative $=$

Fréquence par espèce

Fréquence totale de toutes les espèces $x 100$

- Indice de Valeur d'Importance (IVI) = Dominance relative + Densité relative + Fréquence relative

Les espèces ligneuses dominantes des inselbergs ont été déterminées à partir du calcul des IVI. Ainsi toute espèce ligneuse ayant un IVI $\geq 10$ est considérée comme dominante par rapport à une autre de valeur inférieure.

L'impact de l'anthropisation à travers les traces d'exploitation humaine sur les ligneux. Pour ce faire nous avons affecté le code 1 à tout individu ligneux portant une trace d'exploitation (coupe; écorçage; déracinement; cueillette de feuilles, de fruits).

Le renouvellement des peuplements ligneux des inselbergs a été évalué par le calcul de la proportion des individus juvéniles (individus ayant un diamètre $<5 \mathrm{~cm}$ ) par rapport aux individus adultes (individus ayant un diamètre $\geq 5 \mathrm{~cm}$ ). Les individus juvéniles et adultes ont été dénombrés dans chaque placeau.

Des analyses univariées (Anova de Kruskal-Wallis) ont été utilisées pour comparer les indices de diversité, la densité et la surface terrière en fonction de la nature du substrat et la position topographique (versant, sommet) des inselbergs et entre les secteurs phytogéographiques. Ces analyses ont été effectuées avec le logiciel Minitab 16-2.

\section{Resultats}

\subsection{Dominance des différents types d'inselbergs}

Les inselbergs dominants dans les différents secteurs phytogéographiques sont les inselbergs granitiques suivis par les inselbergs gréseux. Par contre les inselbergs gneissiques n'ont pas été rencontrés le long de notre transect.

\subsection{Composition floristique et diversité spécifiqu}

Les relevés effectués sur les inselbergs du secteur phytogéographique nord-sahélien ont permis de déterminer 37 espèces ligneuses réparties en 14 familles et 24 genres (Tableau 1). Commiphora africana, Pterocarpus lucens, Acacia laeta, Guiera senegalensis, Acacia tortilis, Maerua crassifolia, Balanites aegyptiaca et Combretum micranthum sont les espèces dominantes par ordre d'importance de leurs IVI (Tableau 2). La famille des Fabaceae-Mimosoideae est la plus représentée (18\%) suivie par les Capparaceae (13\%), les Apocynaceae (11\%) et les Malvaceae (11\%) (Tableau $3)$. Le nombre moyen d'espèces par relevé, l'indice de diversité de Shannon, la diversité maximale et l'équitabilité de Piélou sont respectivement $8,07 \pm 2,25$ espèces par relevé, $2,42 \pm 0,44$ bits, 3,37et 0,72 $\pm 0,03$. L'indice de diversité de Shannon indique une forte diversité sur les inselbergs de ce secteur car sa valeur $(2,42)$ se rapproche beaucoup à celle de l'indice de diversité maximale (3,37). L'indice d'équitabilité de Piélou étant moyen indique la dominance de certaines espèces. Ces résultats montrent une bonne répartition des espèces en termes de recouvrement. En considérant la stratification, les versants des inselbergs abritent toutes les 37 espèces recensées, alors que sur les sommets on rencontre 27 espèces (Tableau 1). Les microphanérophytes avec un spectre brut de $54 \%$ et un spectre pondéré de $58 \%$ sont les types biologiques les plus abondants de la végétation ligneuse. Ils sont suivis par les nanophanérophytes avec un spectre brut de $38 \%$ et un spectre pondéré de $23 \%$ (Fig. 7). On note une absence des mésophytes, des chaméphytes et les géophytes sur les inselbergs de ce secteur. Les spectres brut et pondéré des types phytogéographiques montrent une prédominance des espèces soudano-zambéziennes (46\%, $37 \%$ ), suivi des sahélo-soudaniennes (24\%, $27 \%$ ) (Fig. 8).

53 espèces ligneuses ont été recensées sur les inselbergs du secteur sud-sahélien. Ces espèces sont réparties dans 19 familles et 34 genres (Tableau 1). Les espèces dominantes sont Guiera senegalensis, Acacia seyal, Combretum micranthum, Sterculia setigera, Anogeissus leiocarpa, Balanites aegyptiaca et Lannea microcarpa (Tableau 2). Les Fabaceae-Mimosoideae (23\%), les Combretaceae (11\%), les Capparaceae $(9 \%)$ et les Fabaceae-Caesalpinoideae (9\%) sont les familles qui dominent sur les inselbergs de ce secteur. Le nombre moyen d'espèces par relevé est de 7,47 $\pm 3,36$ espèces. L'indice de diversité de Shannon, l'équitabilité de Piélou et la diversité maximale sont respectivement 2,39 \pm 0,09 bits $0,66 \pm 0,02$ et 3,61 (Tableau 1). Les versants des inselbergs de ce secteur sont plus diversifiés que leurs sommets. En effet, c'est sur les versants que se rencontre le plus grand nombre d'espèces, de genres et de familles. L'indice de diversité de Shannon pour les versants se rapproche beaucoup plus à l'indice de diversité maximale. Les types biologiques sont dominés par les microphanérophytes $(70 \%$, $57 \%$ ) et les nanophanérophytes $(25 \%, 41 \%)$ (Fig. 9). On note une absence des mésophytes et des géophytes. Les espèces de liaison soudano-zambézienne sont les plus abondantes $(47 \% ; 89 \%)$ viennent en suite les espèces de la liaison sahélo-soudaniennes (11\%, 3 \%) (Fig. 10).

Les espèces ligneuses inventoriées sur les inselbergs du secteur nord-soudanien sont au nombre de 76 et se répartissent dans 28 familles et 57 genres. Les espèces ligneuses qui dominent sur les inselbergs de ce secteur sont respectivement Detarium microcarpum, Bombax costatum, Sterculia setigera, Stereospermum kunthianum, Boswellia dalzielii, Lannea microcarpa, Vitellaria paradoxa et Lannea acida (Tableau 2). Les familles dominantes sont les Combretaceae $(14 \%)$, les Fabaceae-Mimosoideae (14\%) et les Fabaceae-Caesalpinoideae (11\%). Sur un total de 76 espèces ligneuses recensées 59 espèces se rencontrent sur versants contre 31 espèces pour les sommets (Tableau 1). Les types biologiques dominants sont les microphanérophytes (73\%, $92 \%)$ et nanophanérophytes $(13 \%, 1 \%)$ (Fig. 11). Dans 
Tableau 1: Diversité floristique des inselbergs / inselberg floristic diversity.

$\begin{array}{llllllll}\begin{array}{l}\text { Secteurs phyto- } \\ \text { géographiques }\end{array} & \text { Familles } & \text { Genres } & \text { Espèces } & \text { Nm } & \text { H } & \text { E } & \text { Hmax } \\ \text { Nord-sahélien } & 14 & 24 & 37 & 8,07 \pm 2,25 & 2,42 \pm 0,44 & 0,72 \pm 0,03 & 3,37 \\ \text { Sud-sahélien } & 19 & 34 & 53 & 7,47 \pm 3,36 & 2,39 \pm 0,09 & 0,66 \pm 0,02 & 3,61 \\ \text { Nord-soudanien } & 28 & 57 & 76 & 12,54 \pm 3,14 & 2,68 \pm 0,07 & 0,69 \pm 0,02 & 3,87 \\ \text { Sud-soudanien } & 34 & 89 & 113 & 13,04 \pm 5,59 & 2,56 \pm 0,06 & 0,60 \pm 0,01 & 4,25\end{array}$

Nm: richesse spécifique moyenne par placeau, H: indice de diversité de Shannon, E: indice d'équitabilité de Piélou, Hmax: indice de diversité maximale.

Tableau 2: Indices de Valeurs d'Importances (IVI) des espèces dominantes des inselbergs / importance value index (IVI) of inselberg dominant species.

\begin{tabular}{|c|c|c|c|c|}
\hline Espèces & nord-sahélien & sud-sahélien & nord-soudanien & sud-soudanien \\
\hline Acacia laeta & 27,52 & 4,82 & & \\
\hline Acacia tortilis & 17,47 & 1,66 & & \\
\hline Acacia seyal & 0,31 & 33,27 & 0,37 & \\
\hline Anogeissus leiocarpa & & 13,20 & 7,20 & 3,12 \\
\hline Annona senegalensis & & & 1,01 & 14,26 \\
\hline Balanites aegyptiaca & 13,52 & 11,62 & 3,36 & \\
\hline Bombax costatum & & & 29,85 & 3,74 \\
\hline Boswellia dalzielii & & 9,42 & 13,34 & - \\
\hline Burkea africana & & & 0,86 & 16,27 \\
\hline Combretum micranthum & 12,91 & 39,31 & 4,39 & \\
\hline Commiphora africana & 66,29 & 3,53 & & \\
\hline Detarium microcarpum & & & 67,07 & 4,51 \\
\hline Guiera senegalensis & 26,31 & 72,33 & 2,75 & 2,68 \\
\hline Lannea acida & & 3,42 & 12,51 & 12,11 \\
\hline Lannea microcarpa & 0,91 & 10,85 & 13,29 & 7,29 \\
\hline Leptadenia hastata & 10,88 & & & \\
\hline Maerua crassifolia & 15,10 & & & \\
\hline Pteleopsis suberosa & & & 1,54 & 10,56 \\
\hline Pterocarpus erinaceus & & 4,03 & 3,29 & 15,14 \\
\hline Pterocarpus lucens & 42,04 & & & \\
\hline Sterculia setigera & & 16,75 & 14,17 & 5,46 \\
\hline Stereopermum kunthianum & & 1,40 & 27,17 & \\
\hline Terminalia laxiflora & & & 6,10 & 34,36 \\
\hline Vitelaria paradoxa & & 8,37 & 12,63 & 4,54 \\
\hline
\end{tabular}

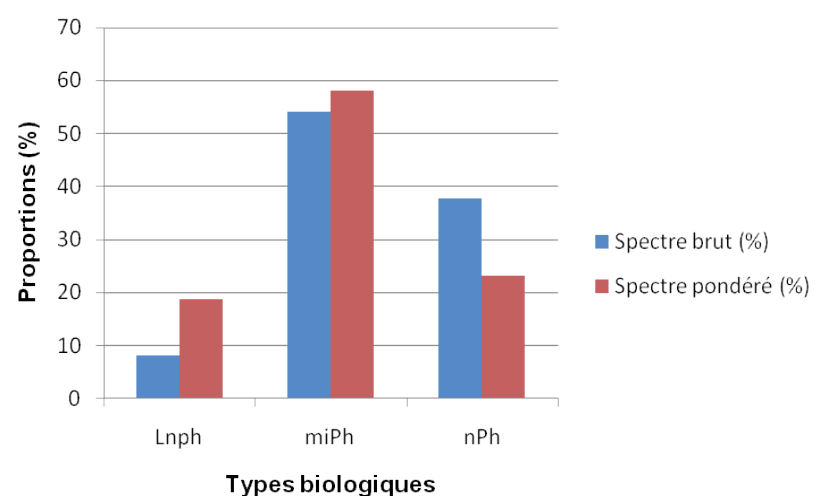

Fig. 7: Spectre des types biologiques des inselbergs du secteur nordsahélien / life form spectrum found of North-Sahelian inselbergs.

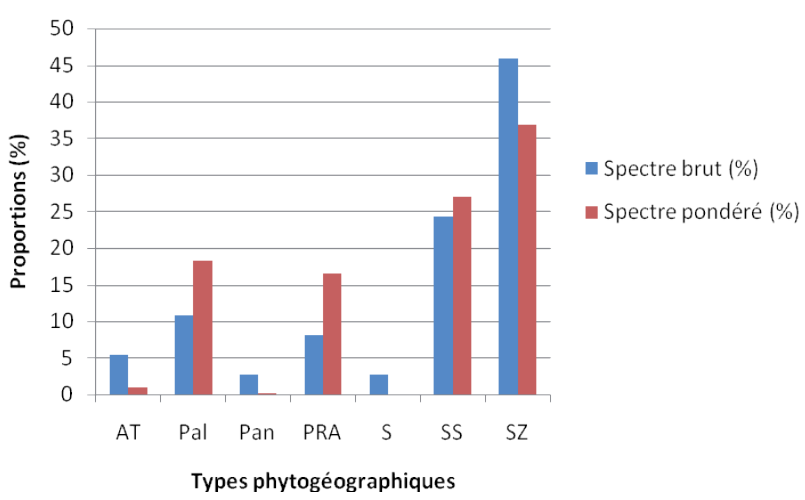

Fig. 8: Spectre des types phytogéographiques des inselbergs du secteur nord-sahélien / phytogeographic spectrum of northsahelian inselbergs. 


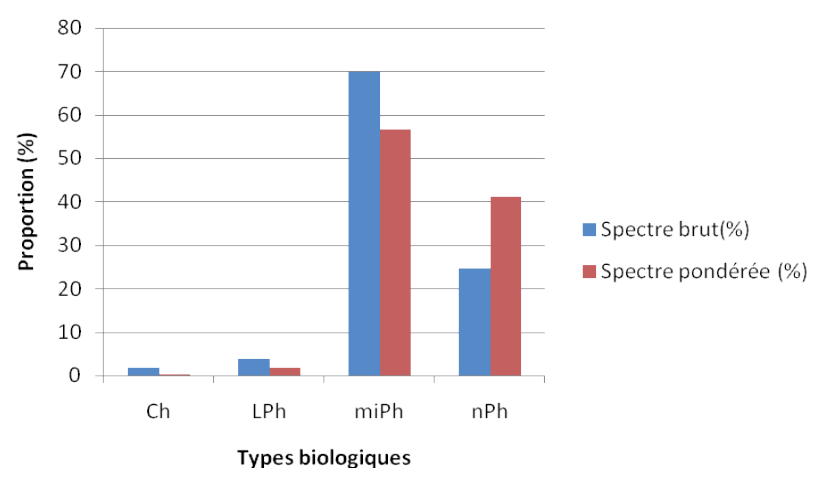

Fig. 9: Spectre des types biologiques des inselbergs du secteur sudsahélien / life form spectrum of South-Sahelian inselbergs.

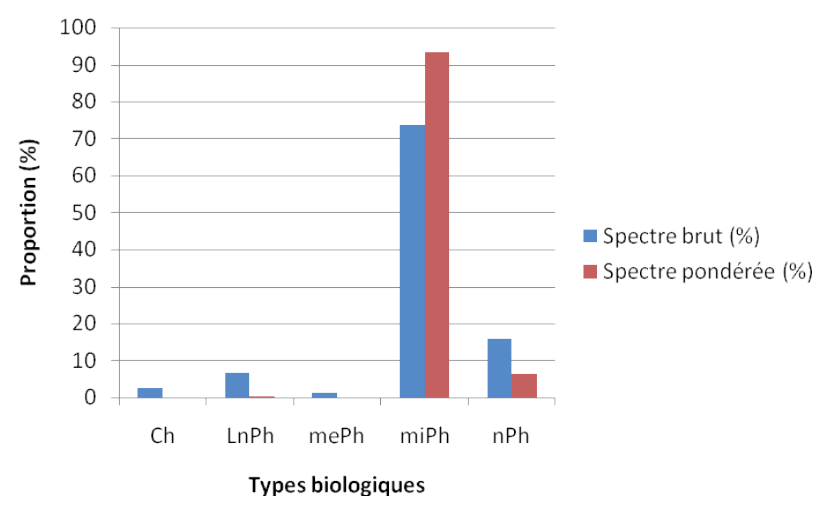

Fig. 11: Spectre des types biologiques du secteur nord-soudanien / life form spectrum of North-Sudanian inselbergs.

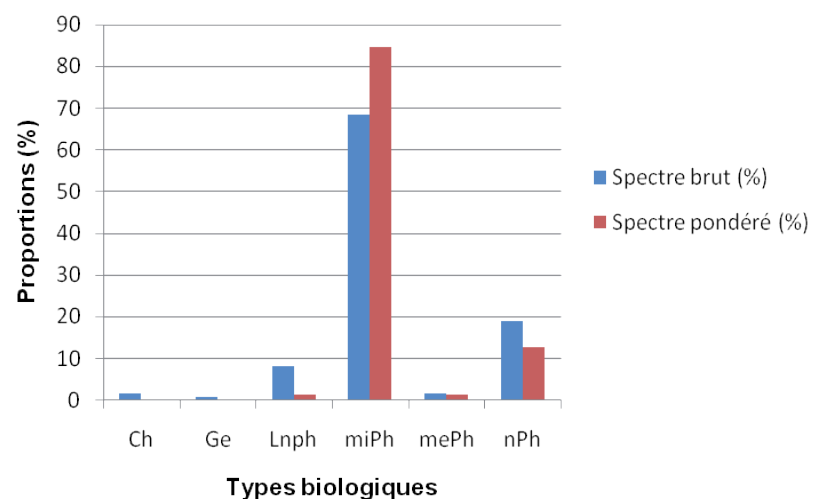

Fig. 13: Spectre des types biologiques des inselbergs du secteur sudsoudanien / life form spectrum of South-Sudanian inselbergs.

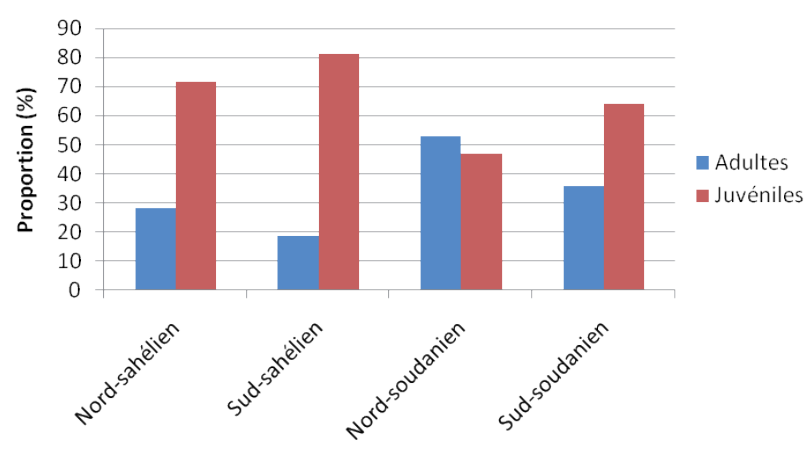

Secteurs phytogéographiques

Fig. 15: Proportion des individus juvéniles dans les peuplements ligneux des inselbergs / proportion of juvenile individuals of woody species on inselbergs.

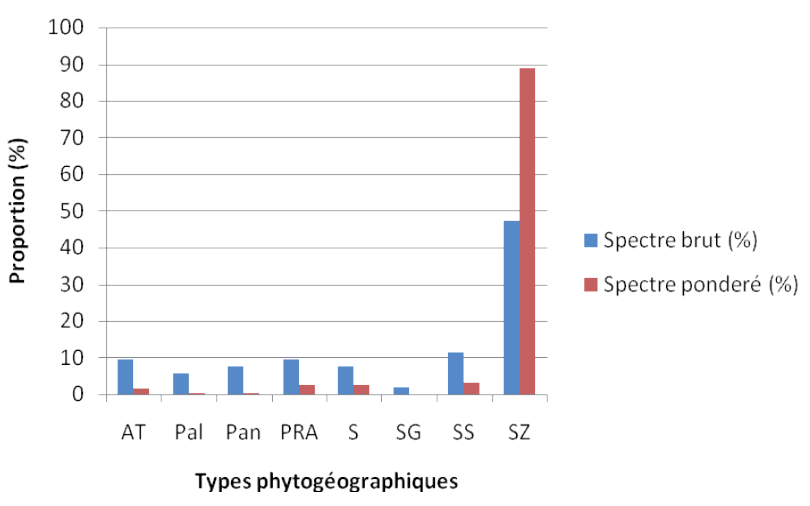

Fig. 10: Spectre des types phytogéographiques des inselbergs du secteur sud-sahélien / phytogeographic spectrum of southsahelian inselbergs.

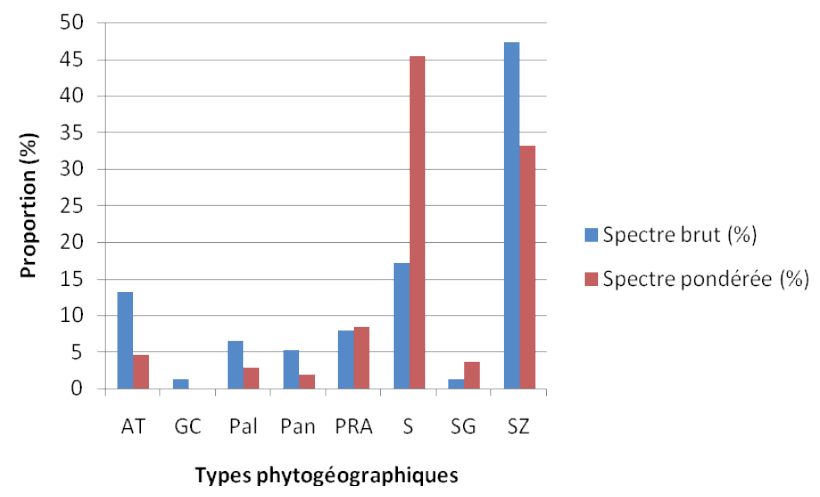

Fig. 12: Spectre des types phytogéographiques du secteur nord-soudanien / phytogeographic spectrum of North-Sudanian inselbergs.

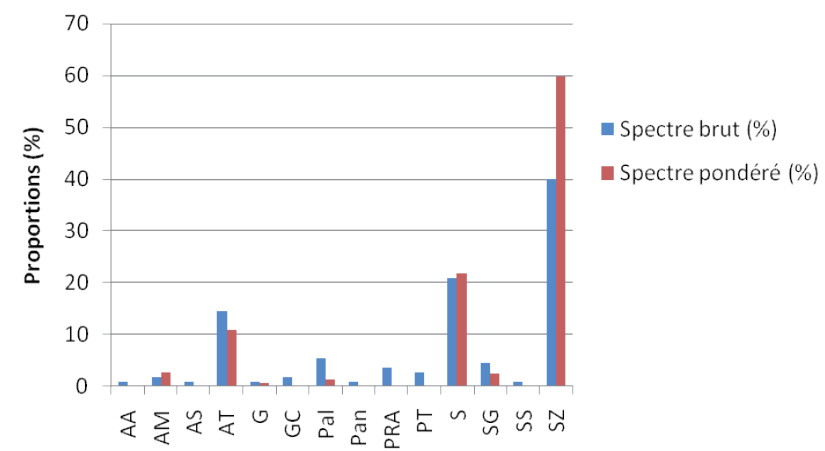

Types phytogéographiques

Fig. 14: Spectre des types phytogéographiques des inselbergs du secteur sud-soudanien / phytogeographic spectrum of SouthSudanian inselbergs. 
Tableau 3: Familles des espèces ligneuses rencencées sur les inselbergs / woody species families from inselbergs.

\begin{tabular}{|c|c|c|c|c|}
\hline Familles & nord-sahélien & sud-sahélien & nord-soudanien & sud-soudanien \\
\hline Anacardiaceae & 7,89 & 7,55 & 3,95 & 6,14 \\
\hline Annonaceae & - & - & 1,32 & 1,75 \\
\hline Apocynaceae & 10,53 & 1,89 & 5,27 & 7,01 \\
\hline Arecaceae & - & - & - & 2,63 \\
\hline Zygophyllaceae & 2,63 & 1,89 & 1,32 & 0,88 \\
\hline Bignoniaceae & - & 1,89 & 2,63 & 0,88 \\
\hline Bixaceae & - & - & 1,32 & 0,88 \\
\hline Burseraceae & 2,63 & 3,77 & 2,63 & - \\
\hline Capparaceae & 13,16 & 9,43 & 1,32 & - \\
\hline Chrysobalanaceae & - & - & - & 1,75 \\
\hline Combretaceae & 10,53 & 11,32 & 14,47 & 11,40 \\
\hline Dipterocarpaceae & - & - & - & 0,88 \\
\hline Ebenaceae & - & 1,89 & 1,32 & 0,88 \\
\hline Euphorbiaceae & 5,26 & 1,89 & 3,95 & 3,51 \\
\hline Salicaceae & - & - & - & 0,88 \\
\hline Lamiaceae & - & - & 2,64 & 2,63 \\
\hline $\begin{array}{l}\text { Fabaceae-Caesalpino- } \\
\text { ideae }\end{array}$ & 5,26 & 9,43 & 10,53 & 7,89 \\
\hline $\begin{array}{l}\text { Fabaceae-Mimosoi- } \\
\text { deae }\end{array}$ & 18,42 & 22,64 & 14,47 & 9,65 \\
\hline Fabaceae-Faboideae & 5,26 & 5,66 & 6,58 & 6,14 \\
\hline Loganiaceae & - & - & 2,63 & 1,75 \\
\hline Meliaceae & - & 1,89 & 1,32 & 1,75 \\
\hline Menispermaceae & 2,63 & - & - & - \\
\hline Moraceae & - & - & 3,95 & 3,51 \\
\hline Ochnaceae & - & - & - & 0,88 \\
\hline Polygalaceae & - & - & 1,32 & 0,88 \\
\hline Phyllantaceae & - & - & 2,63 & 1,75 \\
\hline Rhamnaceae & 2,63 & 1,89 & 2,63 & 1,75 \\
\hline Rubiaceae & 2,63 & 2,63 & 5,66 & 10,52 \\
\hline Rutaceae & - & - & - & 0,88 \\
\hline Sapindaceae & - & - & 1,32 & 0,88 \\
\hline Sapotaceae & - & - & 1,32 & 2,63 \\
\hline Simaroubaceae & - & - & - & 0,88 \\
\hline Sterculiaceae & - & 1,89 & 1,32 & 0,88 \\
\hline Malvaceae & 10,53 & 5,66 & 3,95 & 5,26 \\
\hline Vitaceae & - & - & 1,32 & 0,88 \\
\hline Ximeniaceae & - & 1,89 & 1,32 & 0,88 \\
\hline
\end{tabular}

Code: - = absence

ce secteur nous notons également l'absence des géophytes sur les inselbergs. Les types phytogéographiques sont dominés par les espèces de la liaison soudano-zambézienne (48 $\%, 34 \%$ ) suivis par les espèces de la liaison Soudanienne $(17 \%, 46 \%)$ (Fig. 12). Les versants regorgent le plus grand nombre d'espèces comparativement aux sommets des inselbergs.

Un total de 113 espèces ligneuses a été recensé sur les inselbergs du secteur sud-soudanien. Ces espèces sont réparties en 89 genres et 34 familles (Tableau 1). Les espèces dominantes sont par ordre d'importance, Terminalia laxiflora, Burkea africana, Pterocarpus erinaceus, Annona senegalensis, Lannea acida et Pteleopsis suberosa (Tableau 2). Les familles dominantes sont les Combretaceae (11\%), les Rubiaceae (11\%) et les Fabaceae-Mimosoideae (10\%), (Tableau 3). Le nombre moyen d'espèces par relevé, l'indice de diversité de Shannon, la diversité maximale et l'équitabilité de Piélou sont respectivement, 13,04 \pm 5,59 espèces par re- 
levé, 2,56 $\pm 0,06$ bits, 4,25 et 0,60 $\pm 0,01$ (Tableau 1). La diversité de Shannon indique une bonne diversité sur les inselbergs du secteur sud-soudanien et la faible valeur de l'indice d'équitabilité de Piélou illustre la dominance de certaines espèces sur les autres. Ces indices montrent une répartition irrégulière des espèces en termes de recouvrement. La répartition irrégulière des espèces est plus remarquable quand on considère les versants et les sommets. Les espèces se rencontrent beaucoup plus sur les versants que sur les sommets. Les types biologiques les plus abondants de ce secteur phytogéographiques sont par ordre d'importance les microphanérophytes $(68 \% ; 84 \%)$, suivis des nanophanérophytes (19 $\% ; 13 \%)$ et les lianes nanophanérophytes $(8 \% ; 1 \%)$. Toute fois, on note la présence des mésophanérophytes, des chaméphytes et des géophytes (Fig. 13). Les spectres phytogéographiques (Fig. 14) présentent une abondance des espèces soudano-zambéziennes $(40 \%, 60 \%)$ et des espèces soudaniennes $(21 \% ; 22 \%)$. Fig. 13 montre également une très faible proportion des espèces Sahélo-sahariennes $(1 \%,<<1$ $\%)$.

\subsection{Les espèces spécificité aux inselbergs de chaque secteur phytogéographique}

Les espèces ligneuses rencontrées uniquement sur les inselbergs des secteurs nord-Sahélien, sud-sahélien, nord-soudanien et sud-soudanien sont respectivement au nombre de 8 espèces, 3 espèces, 4 espèces et 46 espèces. Les espèces ligneuses communes aux inselbergs des quatre secteurs sont au nombre de 13 espèces (Tableau 6). Les indices de similitude de Sørensen (Cs) sont consignés dans le tableau 4 et varient significativement entre les différents secteurs phytogéographiques. Ces indices montrent qu'il y a une similitude, en matière de composition floristique, entre les inselbergs des secteurs nord-sahélien et sud-sahélien ( $\mathrm{Cs}=0,59)$; les inselbergs des secteurs sud-sahélien et nord-soudanien $(\mathrm{Cs}=0,64)$; les inselbergs des secteurs nord-soudanien et sud-soudanien $(\mathrm{Cs}=0,67)$ (Tableau 4).

Tableau 4: Indices de similarité de Sørensen entre inselbergs des différents secteurs phytogéographiques / Sørensen index between inselbergs of differents phytogeographical sectors.

$\begin{array}{llll}\text { sud-sahélien } & \begin{array}{l}\text { nord- } \\ \text { sahélien }\end{array} & \begin{array}{l}\text { sud-sahé- } \\ \text { lien }\end{array} & \begin{array}{l}\text { nord-sou- } \\ \text { danien }\end{array} \\ \begin{array}{l}\text { nord-soudanien } \\ \text { sud-soudanien }\end{array} & 0,35 & 0,64 & \\ & 0,19 & 0,42 & 0,67\end{array}$

La composition floristique ne varie pas significativement en fonction de la nature du substrat des inselbergs. L'indice de Sørensen obtenu $(\mathrm{Cs}=0,70)$ indique une forte similarité entre les inselbergs granitiques et les inselbergs gréseux.

Les sommets et les versants présentent également une forte similarité $(\mathrm{Cs}=0,89)$ en terme de richesse floristique. La majorité des espèces locales inventoriées se rencontrent aussi bien sur les versants que sur les sommets. Ce qui n'est pas le cas des espèces exotiques (Azadirachta indica A. Juss. et Anacardium occidentale L.) qui se rencontre uniquement sur les versants.

\subsection{Etat des peuplements ligneux}

\subsubsection{Paramètres structuraux}

La densité moyenne des ligneux varie significativement suivant les secteurs phytogéographiques $(\mathrm{p}=0,000)$. Elle est de 729,31 $\pm 449,64$ pieds/ha pour le secteur nord-sahélien, $586,05 \pm 186,51 \mathrm{pieds} / \mathrm{ha}$ pour le secteur sud-sahélien, $592,31 \pm 177,10 \mathrm{pieds} / \mathrm{ha}$ pour le secteur nord-soudanien et $863,04 \pm 342,09$ pieds/ha pour le secteur sud-soudanien (Tableau 5). Toute fois on remarque une faible densité moyenne pour les secteurs sud-sahélien et nord-soudanien. Le diamètre moyen des ligneux augmente suivant le gradient climatique. Il est de 10,23 $\pm 5,20$ dans le secteur nordsahélien, 11,67 $\pm 8,27$ dans le secteur sud-sahélien, 16,49 \pm 10,60 dans le secteur nord-soudanien et 16,99 $\pm 11,21$ dans le secteur sud-soudanien. La hauteur moyenne comme le diamètre moyen augmente suivant le gradient. Elle est respectivement de 2,64 $\pm 2,28 ; 2,74 \pm 1,49 ; 3,81 \pm 1,91 ; 4,05$ $\pm 2,26$ dans les secteurs nord-sahélien, sud-sahélien, nordsoudanien et sud-soudanien (Tableau 5). La surface terrière moyenne varie également significativement suivant les secteurs phytogéographiques $(\mathrm{p}=0,000)$. Elle est respectivement de 2,56 $\pm 1,88,1,58 \pm 1,25,8,50 \pm 3,44$ et 3,42 $\pm 3,28$ pour les secteurs nord-sahélien, sud-sahélien, nordsoudanien et sud-soudanien. Les inselbergs sur lesquels on enregistre plus de ligneux portant des traces d'exploitation sont ceux des secteurs sud-sahélien (45\%) et nord-soudanien $(14 \%)$. La variation de la densité moyenne et de la surface terrière moyenne entre les versants et les sommets n'est pas significative $(0,95$ et 0,06 , respectivement pour la densité moyenne et la surface terrière moyenne). Quant au taux d'exploitation, il varie significativement en fonction de la topographie $(\mathrm{p}<0,0001)$. Il est plus faible sur les sommets que sur les versants (Tableau 5).

Tableau 5: Caractéristiques structurales des peuplements ligneux des inselbergs suivant le gradient phytogéographique / structural characteristics of woody population of inselbergs along phytogeographical gradient.

$\begin{array}{lllll}\begin{array}{l}\text { Secteurs phytogéo- } \\ \text { graphiques }\end{array} & \begin{array}{l}\text { Densité moyenne (in- } \\ \text { dividus/ha) }\end{array} & \begin{array}{l}\text { Diamètre moyen } \\ \mathbf{( c m )}\end{array} & \begin{array}{l}\text { Hauteur moyenne } \\ (\mathbf{m})\end{array} & \begin{array}{l}\text { Surface terrière mo- } \\ \text { yenne }\left(\mathbf{m}^{2} / \mathbf{h a}\right)\end{array} \\ \text { Nord-sahélien } & 730 \pm 450 & 10,2 \pm 5,2 & 2,6 \pm 2,3 & 2,6 \pm 1,9 \\ \text { Sud-sahélien } & 586 \pm 187 & 11,7 \pm 8,3 & 2,7 \pm 1,5 & 1,6 \pm 1,3 \\ \text { Nord-soudanien } & 592 \pm 177 & 16,5 \pm 10,6 & 3,8 \pm 1,9 & 8,5 \pm 3,4 \\ \text { Sud-soudanien } & 863 \pm 342 & 17,0 \pm 11,2 & 4,1 \pm 2,3 & 3,4 \pm 3,3\end{array}$




\subsubsection{Renouvellement des peuplements ligneux des insel- bergs}

Les individus juvéniles (toutes espèces confondues) dominent dans les peuplements ligneux des secteurs nordsahélien, sud-sahélien et sud-Soudanien (Fig. 15). Dans le secteur nord-sahélien les individus jeunes représentent $72 \%$ de la population tandis que les individus adultes représentent $28 \%$. Dans la population ligneuse du secteur sud-sahélien les juvéniles représentent $81 \%$ des individus alors que les adultes représentent $19 \%$ des individus. Sur les inselbergs $\mathrm{du}$ secteur sud-soudanien les individus juvéniles dominent dans la population ligneuse (65\%) par rapport aux individus adultes qui représentent $36 \%$ de la population. Par contre dans le secteur nord-soudanien ce sont les individus adultes qui dominent dans la population ligneuse. En effet, dans ce secteur les individus adultes représentent $53 \%$ de la population contre $47 \%$ d'individus juvéniles.

Le calcul de la proportion des individus jeunes et adultes suivant la topographie (versant, sommet) donne les mêmes tendances (Tableau 6). Pour les secteurs nord-sahélien, sudsahélien et sud-soudanien les individus jeunes dominent sur les versants et sur les sommets des inselbergs. On enregistre l'effet contraire pour le secteur nord-soudanien où les individus adultes dominent à tous les nivéaux.

Tableau 6: Densité moyenne (pieds/ha) des individus adultes et des individus juvéniles / mean density (individual/ha) of adult and juvenile individual.

$\begin{array}{lll}\begin{array}{l}\text { Secteurs phytogéogra- } \\ \text { phiques }\end{array} & \text { Adultes } & \text { Juvéniles } \\ \text { Nord-sahélien } & 187 \pm 103 & 478 \pm 428 \\ \text { Sud-sahélien } & 109 \pm 65 & 477 \pm 190 \\ \text { Nord-soudanien } & 315 \pm 147 & 278 \pm 158 \\ \text { Sud-soudanien } & 314 \pm 205 & 566 \pm 197\end{array}$

\section{Discussion}

\subsection{Composition floristique et diversité spécifique}

Les 143 espèces ligneuses recensées sur les inselbergs des quatre secteurs phytogéographiques représentent $65 \%$ des espèces ligneuses et $7 \%$ de la flore totale du Burkina Faso. En effet, le Burkina Faso compte au total 2067 espèces végétales dont 220 espèces ligneuses (Thiombiano et al. 2012). Les inselbergs abritent de ce fait une bonne partie des espèces ligneuses du Burkina Faso. La richesse et diversité floristiques varient significativement entre les secteurs phytogéographiques. Cette variation s'illustre par la variation des indices de diversité de Shannon, d'équitabilité de Piélou ( $\mathrm{p}=0,012, \mathrm{p}=0,001$ respectivement) et de l'indice de diversité maximale (Tableau 1). Anogeissus leiocarpa, Combretum micranthum, Maerua crassifolia, Pterocarpus lucens, Lannea microcarpa et Sterculia setigera, espèces menacées des plaines du domaine sahélien, Bombax costatum, Detarium microcarpum, Boswellia dalzielii, Pterocarpus erinaceus, Sterculia setigera, Stereospermum kunthianum et Vitellaria paradoxa, espèces menacées des plaines du domaine soudanien (Thiombiano \& Kampmann 2010, TraOrÉ et al. 2011), se révèlent dominantes sur les insel- bergs. Cet état de fait exprimerait le caractère refuge des inselbergs pour ces 12 espèces. Mais il faut noter que ces 12 espèces ne sont pas restreintes aux inselbergs, seulement elles subissent moins de pressions anthropiques sur les inselbergs que sur les plaines.

Dans le secteur nord-sahélien, les inventaires floristiques réalisés par Ganaba (1994, 2008) et OuÉDRAOGO (2006), ont permis de recenser respectivement 56,34 et 53 espèces ligneuses pour l'ensemble des écosystèmes exceptés les inselbergs. Le recensement de 37 espèces ligneuses sur les inselbergs au cours de cette étude, montre que ces écosystèmes renferment une tranche très importante de la composition floristique de la végétation ligneuse du Sahel strict. Les mêmes auteurs ont mentionné également la dominance des Fabaceae-Mimosoideae, des Capparaceae, des Asclepiadaceae, des Combretaceae et des Malvaceae dans la partie septentrionale du domaine sahélien. Ces familles seraient donc caractéristiques du domaine sahélien du Burkina Faso. Du point de vue physionomique, la végétation des inselbergs du secteur nord-sahélien est dominée par les steppes arbustives où les ligneux dépassent rarement $7 \mathrm{~m}$ de hauteur. Cette physionomie se justifie par la large dominance des microphanérophytes et des nanophanérophytes, et l'absence remarquable des mésophanérophytes et des mégaphanérophytes. En effet, les deux types biologiques (microphanérophytes et nanophanérophytes) représentent $921 \%$ et 81 $\%$ respectivement du spectre brut et du spectre pondéré des types biologiques du secteur nord-sahélien. Pour le spectre phytogéographique, nos résultats sont similaires à ceux de OuÉDRAOGO (2006) qui a aussi souligné la dominance des éléments soudano-zambéziens et sahélo-sahariens.

La dominance des Fabaceae-Mimosoideae, des Combretaceae, des Capparaceae et des Fabaceae-Caesalpinioideae dans les secteurs sud-sahélien et nord-soudanien est en conformité avec ceux de Thiombiano (1996) et de Savadogo et al. 2010. La dominance des Fabaceae-Mimosoideae et des Combretaceae sur les inselbergs de ces secteurs révèle un climat généralement sec (Aubreville 1950). Ce pendant, l'abondance des espèces des liaisons soudano-zambésienne et soudanienne dans le secteur nord-soudanien met en évidence le caractère soudanien de la flore de ce secteur. La prédominance des microphanérophytes et des nanophanérophytes incarne la dominance des savanes dans ces deux secteurs.

Certaines familles dominantes (Rubiaceae, Fabaceae-Caesalpinioideae, Fabaceae-Mimosoideae) du secteur sudsoudanien ont été citées par KouAssi et al. (2009), comme familles dominantes de la végétation des inselbergs du sud-est de la Côte d'Ivoire. Les Rubiaceae et les FabaceaeCaesalpinioideae ont également été cités parmi les familles dominantes des inselbergs du Gabon et du Cameroun respectivement par n'Gok BaNaK (2005) et ViLLIERs (1981). Ces deux familles font partie des principales familles des inselbergs des zones de forêt (KouAssi et al. 2009). La dominance des Rubiaceae et Fabaceae-Caesalpinioideae montre alors la supériorité de l'humidité du secteur sud-soudanien par rapport aux trois autres secteurs. En effet, le secteur sudsoudanien est le secteur le plus humide des secteurs phytogéographiques du Burkina Faso. Il reçoit 900 à 1100 mm d'eau par an et la saison pluvieuse dure 6 à 7 mois Fontès 
Tableau 7: Liste des espèces ligneuses recensées sur les inselbergs / list of woody species inventoried on inselbergs.

$\begin{array}{lllll}\text { Types } & \text { Types } & \text { Familles } & \text { Espèces végétales } & \text { Sahélien } \\ \text { biol. } & \begin{array}{l}\text { phyto- } \\ \text { géogra- } \\ \text { phiques }\end{array} & & \end{array}$

$\begin{array}{lll}\text { miPh } & \mathrm{SZ} & \text { Fabaceae-Mimosoideae } \\ \text { miPh } & \mathrm{SZ} & \text { Fabaceae-Mimosoideae } \\ \text { miPh } & \mathrm{SZ} & \text { Fabaceae-Mimosoideae } \\ \text { miPh } & \mathrm{S} & \text { Fabaceae-Mimosoideae } \\ \text { miPh } & \mathrm{SZ} & \text { Fabaceae-Mimosoideae } \\ \text { miPh } & \mathrm{SZ} & \text { Fabaceae-Mimosoideae } \\ \mathrm{miPh} & \mathrm{SZ} & \text { Fabaceae-Mimosoideae } \\ \mathrm{miPh} & \mathrm{SZ} & \text { Fabaceae-Mimosoideae } \\ \mathrm{miPh} & \mathrm{AT} & \text { Fabaceae-Mimosoideae } \\ \mathrm{miPh} & \mathrm{SS} & \text { Fabaceae-Mimosoideae } \\ \mathrm{miPh} & \mathrm{S} & \text { Malvaceae } \\ \mathrm{miPh} & \mathrm{SZ} & \text { Fabaceae-Caesalpinoideae } \\ \mathrm{nPh} & \mathrm{AT} & \text { Sapindaceae } \\ \mathrm{mPh} & \mathrm{PT} & \text { Anacardiaceae } \\ \mathrm{mPh} & \mathrm{S} & \text { Annonaceae } \\ \mathrm{nPh} & \mathrm{SZ} & \text { Combretaceae } \\ \mathrm{miPh} & \mathrm{SZ} & \text { Meliaceae } \\ \mathrm{miPh} & \mathrm{SG} & \text { Apocynaceae } \\ \mathrm{miPh} & \mathrm{SZ} & \text { Zygophyllaceae } \\ \mathrm{miPh} & \mathrm{SZ} & \text { Fabaceae-Caesalpinoideae } \\ \mathrm{miPh} & \mathrm{S} & \text { Malvaceae } \\ \mathrm{miPh} & \mathrm{SZ} & \text { Arecaceae }\end{array}$

$\begin{array}{lll}\mathrm{mPh} & \mathrm{SS} & \text { Capparaceae } \\ \mathrm{miPh} & \mathrm{SS} & \text { Capparaceae } \\ \mathrm{miPh} & \mathrm{PRA} & \text { Burseraceae } \\ \mathrm{miPh} & \mathrm{PRA} & \text { Rubiaceae }\end{array}$

$\begin{array}{lll}\text { miPh } & \mathrm{S} & \text { Phyllanthaceae } \\ \mathrm{miPh} & \mathrm{AT} & \text { Fabaceae-Caesalpinoideae } \\ \mathrm{nPh} & \mathrm{SZ} & \text { Capparaceae } \\ \mathrm{nPh} & \mathrm{Pal} & \text { Apocynaceae } \\ \mathrm{LnPh} & \mathrm{Pan} & \text { Capparaceae }\end{array}$

$\begin{array}{lll}\mathrm{miPh} & \mathrm{SZ} & \text { Apocynaceae } \\ \mathrm{miPh} & \mathrm{AT} & \text { Fabaceae-Caesalpinoideae } \\ \mathrm{nPh} & \mathrm{PRA} & \text { Fabaceae-Faboideae } \\ \mathrm{miPh} & \mathrm{Pan} & \text { Malvaceae } \\ \mathrm{nPh} & \mathrm{S} & \text { Vitaceae } \\ \mathrm{miPh} & \mathrm{SZ} & \text { Bixaceae } \\ \mathrm{miPh} & \mathrm{SZ} & \text { Combretaceae } \\ \mathrm{miPh} & \mathrm{S} & \text { Combretaceae } \\ & & \\ \mathrm{miPh} & \mathrm{S} & \text { Combretaceae } \\ \mathrm{miPh} & \mathrm{SZ} & \text { Combretaceae }\end{array}$

Acacia ataxacantha DC.

Acacia dudgeonii Craib. ex Hall.

Acacia erythrocalyx Brenan

Acacia gourmaensis A. Chev.

Acacia laeta R. Br. ex Benth.

Acacia macrostachya Reichenb. ex DC.

Acacia nilotica (L.) Willd. ex. Del.

Acacia seyal Del.

Acacia sieberiana DC.

Acacia tortilis (Forssk.) Hayne

Adansonia digitata $\mathrm{L}$.

Afzelia africana SM.

Allophylus africanus P. Beauv.

Anacardium occidentale L.

Annona senegalensis Pers.

Anogeissus leiocarpa (DC.) Guill.\& Perr.

Azadirachta indica A. Juss.

Baissea multiflora A. DC.

Balanites aegyptiaca (L.) Del.

Bauhinia rufescens Lam.

Bombax costatum Pellegr. \& Vuillet.

Borassus akeassi Bayton, Ouedraogo \& Guinko

Boscia angustifolia A. Rich.

Boscia senegalensis (Pers.) Lam. Ex Poir.

Boswellia dalzielii Hutch.

Breonadia salinicina (Vahl) Hepper \&

Wood.

Bridelia scleroneura Müll. \& Arg.

Burkea africana Hook.f.

Cadaba glandulosa Forsk.

Calotropis procera (Ait.) Ait. f.

Capparis sepiaria L. var. fisheri (Pax) De Wolf.

Desmidorchis acutangula Decne

Cassia sieberiana DC.

Cassia singueana Lam.

Ceiba pentandra (L.) Gaertn

Cissus populnea Guill. \& Perr.

Cochlospermum planchonii Hook.f.

Combretum aculeatum Vent.

Combretum adenogonium Steud. ex A.Rich.

Combretum collinum Fresen.

Combretum glutinosum Perr. ex. DC. nord- sud- nord- sud-

\begin{tabular}{|c|c|c|}
\hline+ & + & + \\
\hline - & - & + \\
\hline - & + & + \\
\hline - & + & - \\
\hline+ & + & - \\
\hline+ & + & + \\
\hline+ & + & + \\
\hline+ & + & + \\
\hline - & + & + \\
\hline+ & + & - \\
\hline - & - & + \\
\hline - & - & - \\
\hline - & - & + \\
\hline - & - & - \\
\hline - & - & + \\
\hline - & + & + \\
\hline - & + & + \\
\hline - & - & - \\
\hline+ & + & + \\
\hline+ & + & - \\
\hline - & - & + \\
\hline - & - & - \\
\hline+ & + & - \\
\hline+ & + & - \\
\hline - & + & + \\
\hline - & - & - \\
\hline - & - & + \\
\hline - & - & + \\
\hline+ & - & - \\
\hline+ & - & - \\
\hline - & + & - \\
\hline+ & - & - \\
\hline - & + & + \\
\hline - & + & + \\
\hline - & - & - \\
\hline - & - & + \\
\hline - & - & + \\
\hline+ & + & + \\
\hline - & - & - \\
\hline - & - & + \\
\hline+ & + & + \\
\hline
\end{tabular}




\begin{tabular}{|c|c|c|c|c|}
\hline $\begin{array}{l}\text { Types } \\
\text { biol. }\end{array}$ & $\begin{array}{l}\text { Types } \\
\text { phyto- } \\
\text { géogra- } \\
\text { phiques }\end{array}$ & Familles & Espèces végétales & Sahélien \\
\hline
\end{tabular}

$\begin{array}{lll}\text { miPh } & \text { SZ } & \begin{array}{l}\text { Combretaceae } \\ \text { miPh }\end{array} \\ \text { miPh } & \text { ST } & \begin{array}{l}\text { Combretaceae } \\ \text { Combretaceae }\end{array} \\ \text { miPh } & \text { SZ } & \text { Burseraceae } \\ \text { miPh } & \text { PRA } & \text { Fabaceae-Faboideae } \\ & & \text { Rubiaceae } \\ \text { nPh } & \text { AT } & \text { Euphorbiaceae } \\ \text { LnPh } & \text { SZ } & \text { Fabaceae-Caesalpinoideae } \\ \text { miPh } & \text { S } & \text { Fabaceae-Faboideae } \\ \text { miPh } & \text { SZ } & \text { Fabaceae-Caesalpinoideae } \\ \text { miPh } & \text { SZ } & \text { Fabaceae-Caesalpinoideae } \\ \text { nPh } & \text { S } & \text { Fabaceae-Mimosoideae } \\ \text { miPh } & \text { AT } & \text { Ebenaceae } \\ \text { miPh } & \text { SZ } & \end{array}$

$\begin{array}{lll}\operatorname{miPh} & \mathrm{GC} & \text { Arecaceae } \\ \mathrm{miPh} & \mathrm{SZ} & \text { Fabaceae-Mimosoideae } \\ \mathrm{miPh} & \mathrm{SG} & \text { Fabaceae-Faboideae }\end{array}$

$\begin{array}{lll}\mathrm{Ch} & \mathrm{SZ} & \text { Euphorbiaceae } \\ \mathrm{nPh} & \mathrm{S} & \text { Rubiaceae } \\ \mathrm{mPh} & \text { At } & \text { Fabaceae-Mimosoideae }\end{array}$

$\mathrm{mPh}$ At Fabaceae-Mimosoideae

$\mathrm{miPh} \quad \mathrm{SZ} \quad$ Rubiaceae

$\mathrm{miPh} \quad \mathrm{S} \quad$ Moraceae

miPh SZ Moraceae

miPh PRA Moraceae

$\mathrm{miPh} S \mathrm{~S} \quad$ Moraceae

miPh PRA Moraceae

miPh SZ Salicaceae

$\mathrm{nPh} \quad$ Pal Phyllanthaceae

$\mathrm{nPh} \quad \mathrm{S} \quad$ Rubiaceae

$\mathrm{nPh} \quad \mathrm{S} \quad$ Rubiaceae

$\mathrm{nPh} \quad \mathrm{Pal} \quad$ Rubiaceae

$\mathrm{miPh} \quad \mathrm{SZ} \quad$ Malvaceae

$\mathrm{miPh} S \mathrm{~S} \quad$ Malvaceae

$\mathrm{nPh} \quad \mathrm{Pal} \quad$ Malvaceae

$\mathrm{miPh} S \mathrm{~S} \quad$ Malvaceae

$\mathrm{miPh} \quad \mathrm{SS} \quad$ Malvaceae

$\mathrm{nPh} \quad \mathrm{SS} \quad$ Malvaceae

miPh SZ Combretaceae

miPh SZ Fabaceae-Caesalpinoideae

miPh SG Simaroubaceae

$\mathrm{miPh}$ AT Apocynaceae

miPh SZ Phyllanthaceae

miPh S Fabaceae-Caesalpinoideae
Combretum micranthum G. Don.

Combretum molle R. Br. ex G. Don

Combretum nigricans Lepr. ex Guill. \& Perr.

Commiphora africana (A. Rich.) Engl.

Cordyla pinnata (Lepr.ex A.Rich.) MilneRedh.

Crossopteryx febrefuga (Afzel. ex G.Don) Benth.

Croton pseudopulchellus Pax

Cynanchum hastifolium N. E. Br. Dalbergia melanoylon Guill. \& Perr.

Daniellia oliveri (Rolfe) Hutch. et Dalz.

Detarium microcarpum Guill \& Perr.

Dichrostachys cinerea (L.) Wight \& Am.

Diospyros mespiliformis Hochst. ex A.

DC.

Elaeis guineensis Jacq.

Entada africana Guill. \& Perr.

Erythrina senegalensis DC.

Euphorbia balsamifera Ait.

Fadogia agrestis Schweinf. ex Hiern

Faidherbia albida (Delile) A. Chev.

Feretia apodanthera Del.

Ficus abutilifolia (Miq.) Miq.

Ficus cordat.

Ficus dicranosty Mildbr.

Ficus glumosa Delile

Ficus ingens Miq.

Flacourtia indica (Burm. f.) Merr.

Flueggea virosa (Roxb. ex Willd.) Voigt.

Gardenia erubescens Stapf et Hutch.

Gardenia sokotensis Hutch.

Gardenia ternifolia Schumach. \& Thonn.

Grewia bicolor Juss.

Grewia cissoides Hutch. \& Dalz.

Grewia flavescens Juss.

Grewia lasiodiscus K. Schum.

Grewia tenax (Forssk.) Fiori

Grewia villosa Willd.

Guiera senegalensis J. G. Gmel.

Gymnosporia senegalensis (Lam.) Loes.

Quassia undulata (Guill.\& Perr.) F.Dietr.

Holarrhena floribunda (G. Don) Dur. \& Schinz

Hymenocardia acida Tul.

Isoberlinia doka Craib \& Stapf. nord- sud- nord- sud-

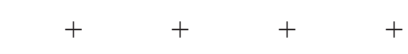




\begin{tabular}{|c|c|c|c|c|}
\hline $\begin{array}{l}\text { Types } \\
\text { biol. }\end{array}$ & $\begin{array}{l}\text { Types } \\
\text { phyto- } \\
\text { géogra- } \\
\text { phiques }\end{array}$ & Familles & Espèces végétales & Sahélien \\
\hline
\end{tabular}

\begin{tabular}{|c|c|c|}
\hline $\mathrm{nPh}$ & Pan & Euphorbiaceae \\
\hline $\mathrm{mePh}$ & $\mathrm{S}$ & Meliaceae \\
\hline $\mathrm{mPh}$ & SZ & Apocynaceae \\
\hline $\mathrm{miPh}$ & $\mathrm{S}$ & Anacardiaceae \\
\hline $\mathrm{miPh}$ & SZ & Anacardiaceae \\
\hline $\mathrm{nPh}$ & G & Anacardiaceae \\
\hline $\mathrm{LnPh}$ & SZ & Apocynaceae \\
\hline $\mathrm{LnPh}$ & SZ & Apocynaceae \\
\hline $\mathrm{miPh}$ & $\mathrm{SZ}$ & Ochnaceae \\
\hline $\mathrm{miPh}$ & AT & Capparaceae \\
\hline $\mathrm{miPh}$ & SZ & Capparaceae \\
\hline $\mathrm{miPh}$ & AT & Sapotaceae \\
\hline $\mathrm{miPh}$ & SZ & Chrysobalanaceae \\
\hline $\mathrm{miPh}$ & $\mathrm{SZ}$ & Rubiaceae \\
\hline $\mathrm{nPh}$ & PRA & Dipterocarpaceae \\
\hline $\mathrm{nPh}$ & SG & Anacardiaceae \\
\hline $\mathrm{miPh}$ & SZ & Sapotaceae \\
\hline $\mathrm{miPh}$ & SZ & Chrysobalanaceae \\
\hline $\mathrm{mePh}$ & $\mathrm{Pal}$ & Fabaceae-Mimosoideae \\
\hline $\mathrm{miPh}$ & PRA & Rubiaceae \\
\hline $\mathrm{miPh}$ & $\mathrm{S}$ & Fabaceae-Faboideae \\
\hline $\mathrm{miPh}$ & SZ & Fabaceae-Caesalpinoideae \\
\hline $\mathrm{miPh}$ & AT & Fabaceae-Caesalpinoideae \\
\hline $\mathrm{miPh}$ & $\mathrm{SZ}$ & Fabaceae-Mimosoideae \\
\hline $\mathrm{miPh}$ & SZ & Combretaceae \\
\hline $\mathrm{miPh}$ & $\mathrm{SZ}$ & Fabaceae-Faboideae \\
\hline $\mathrm{mPh}$ & PRA & Fabaceae-Faboideae \\
\hline $\mathrm{miPh}$ & SZ & Arecaceae \\
\hline $\mathrm{LnPh}$ & S & Rubiaceae \\
\hline $\mathrm{LmPh}$ & AA & Apocynaceae \\
\hline $\mathrm{miPh}$ & $\mathrm{SZ}$ & Apocynaceae \\
\hline $\mathrm{nPh}$ & AT & Euphorbiaceae \\
\hline $\mathrm{miPh}$ & $\mathrm{SZ}$ & Euphorbiaceae \\
\hline $\mathrm{miPh}$ & $\mathrm{SZ}$ & Rubiaceae \\
\hline $\mathrm{nPh}$ & $\mathrm{SZ}$ & Anacardiaceae \\
\hline $\mathrm{miPh}$ & AT & Polygalaceae \\
\hline $\mathrm{miPh}$ & $\mathrm{SZ}$ & Rubiaceae \\
\hline $\mathrm{miPh}$ & SZ & Anacardiaceae \\
\hline $\mathrm{miPh}$ & SZ & Malvaceae \\
\hline $\mathrm{miPh}$ & SG & Bignoniaceae \\
\hline $\mathrm{nPh}$ & $\mathrm{GC}$ & Apocynaceae \\
\hline
\end{tabular}

Jatropha gossypiifolia L.

Khaya senegalensis (Desr.) A. Juss.

Landolphia heudelotii A. DC.

Lannea acida A. Rich.

Lannea microcarpa Engl. \& K. Krause

Lannea velutina A. Rich.

Leptadenia hastata (Pers.) Decne.

Leptadenia pyrotechnica (Forsk.) Decne.

Lophira lanceolata Van Tiech.ex Keay

Maerua angolensis DC.

Maerua crassifolia Forsk.

Manilkara multinervis (Bak.) Dubard

Maranthes polyandra (Benth.)Prance

Mitragyna inermis (Willd.) Kuntze

Monotes kerstingii Gilg

Ozoroa obovata(Oliv.) R.Fern. \& A.Fern.

Pachystela pobeguinianum (Pierre ex Lecomte) Aké Assi \& L.Gaut

Parinari curatellifolia Planch. ex Benth.

Parkia biglobosa (Jacq.) R. Br. Ex. G.

Don

Pavetta corymbosa (DC.) F. N. Williams

Pericopsis laxiflora (Benth.) van Meeuwen

Piliostigma reticulatum (DC.) Hochst.

Piliostigma thonningii (Schumach.) Milne-Redh.

Prosopis africana (Guill. et Perr.) Taub.

Pteleopsis suberosa Engl. et Diels

Pterocarpus erinaceus Poir.

Pterocarpus lucens Guill. \& Perr.

Raphia sudanica A. Chev.

Rytigynia senegalensis Blume

Saba comorensis (Boj.ex DC.) Pichon

Saba senegalensis (A. DC.) Pichon

Shirakiopsis elliptica (Hochst.) Esser

Excoecaria grahamii Stapf

Sarcocephalus latifolius (Sm.) E.A.Bruce

Sclerocarya birrea (A. Rich.) Hochst

Securidaca longepedunculata Fres.

Sericanthe chevalieri (K. Krause) Robbrecht

Sorindeia juglandifolia (A.Rich.) Planch. ex Oliv.

Sterculia setigera Del.

Sterospermum kunthianum Cham.

Strophanthus sarmentosus DC.

nord- sud- nord- sud- 


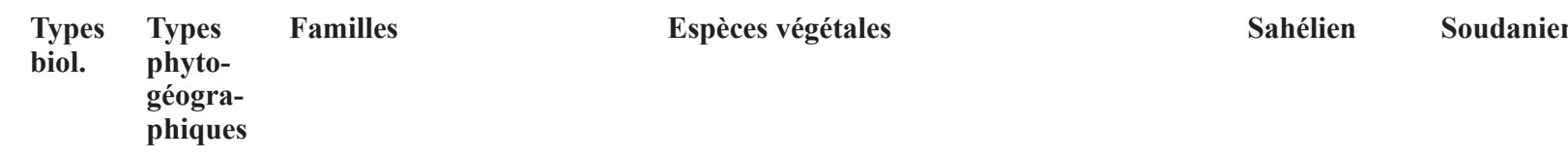

\begin{tabular}{|c|c|c|c|c|c|c|c|}
\hline $\mathrm{miPh}$ & $\mathrm{SZ}$ & Loganiaceae & Strychnos innocua Del. & - & - & + & + \\
\hline $\mathrm{miPh}$ & Pal & Loganiaceae & Strychnos spinosa Lam. & - & - & + & + \\
\hline $\mathrm{nPh}$ & PT & Fabaceae-Caesalpinoideae & Tamarindus indica L. & - & + & + & + \\
\hline $\mathrm{miPh}$ & PRA & Combretaceae & Terminalia avicennioisdes Guill. \& Perr. & - & - & + & - \\
\hline $\mathrm{miPh}$ & AS & Combretaceae & Terminalia catappa $\mathrm{L}$ & - & - & - & + \\
\hline $\mathrm{miPh}$ & $\mathrm{SZ}$ & Combretaceae & Terminalia laxiflora Engl. & - & - & + & + \\
\hline $\mathrm{miPh}$ & $\mathrm{SZ}$ & Combretaceae & Terminalia macroptera Guill. \& Perr. & - & - & + & + \\
\hline $\mathrm{LnPh}$ & $\mathrm{SZ}$ & Lamiaceae & Tinnea barteri Gürke & - & - & + & + \\
\hline $\mathrm{LnPh}$ & PRA & Menispermaceae & Tinospora bakis (A. Rich.) Miers & + & - & - & - \\
\hline $\mathrm{miPh}$ & PRA & Annonaceae & Uvaria chamae P. Beauv & - & - & - & + \\
\hline $\mathrm{miPh}$ & $\mathrm{S}$ & Sapotaceae & Vitellaria paradoxa Gaertn. f. & - & + & + & + \\
\hline $\mathrm{miPh}$ & AT & Lamiaceae & Vitex chrysocarpa Planch. ex Benth. & - & - & + & + \\
\hline $\mathrm{miPh}$ & AT & Lamiaceae & Vitex doniana Sweet & - & - & - & + \\
\hline $\mathrm{nPh}$ & $\mathrm{SZ}$ & Fabaceae-Faboideae & $\begin{array}{l}\text { Xeroderris stuhlmannii (Taub.) Mença \& } \\
\text { E. P. }\end{array}$ & - & - & + & + \\
\hline $\mathrm{miPh}$ & PT & Ximeniaceae & Ximenia americana $\mathrm{L}$ & - & + & + & + \\
\hline $\mathrm{miPh}$ & $\mathrm{SZ}$ & Rutaceae & $\begin{array}{l}\text { Zanthoxylum zantholoides (Lam.) Water- } \\
\text { manu }\end{array}$ & - & - & - & + \\
\hline $\mathrm{miPh}$ & AT & Rhamnaceae & Ziziphus abyssinica A. Rich. & - & - & - & + \\
\hline $\mathrm{miPh}$ & Pal & Rhamnaceae & Ziziphus mauritiana Lam. & + & + & + & - \\
\hline $\mathrm{miPh}$ & PRA & Rhamnaceae & Ziziphus mucronata Willd. & - & + & + & + \\
\hline
\end{tabular}

AA: Afro-américaines, AM: Afro-malgaches, AT: Afro-tropicales, Cos: Cosmopolites, GC: Guinée-congolaises, Pal: Paléotropicales, Pan: Pantropicales, PRA: Plurirégionales africaines, SG: Soudano-guinéennes, SZ: Soudano-Zambéziennes.

MPh: mégaphanérophytes; mePh: mésophanérophytes ; miPH: microphanérophytes; nph: nanophanérophytes; Lnph: phanérophytes lianescentes.

Code: $+=$ Présence; - = Absence

\& Guinko (1995). Cette humidité est confirmée par la proportion élevée des espèces soudaniennes et soudano-zambéziennes. Aké_Assi (2002) rapporte que les régions les plus humides sont les zones de prédilection des Rubiaceae.

La variation de la richesse floristique des inselbergs est beaucoup plus liée aux conditions climatiques qu'aux facteurs topographie et nature du substrat. Les indices de similarité de Sorensen l'ont confirmé. Par ailleurs, les indices de similarité de Sorensen obtenus pour le nord-sahélien et le sudsoudanien $(\mathrm{Cs}=0,19)$, le sud-sahélien et le sud-soudanien $(\mathrm{Cs}=0,42)$ mettent en exergue la dominance du facteur climat dans la richesse floristique.

\subsection{Etat des peuplements ligneux}

La faible densité des ligneux dans les secteurs sud-sahélien et nord-soudanien est liée à la faible altitude des inselbergs qui favorise l'accès à la végétation ligneuse. Les hauteurs moyennes de ces deux secteurs sont respectivement $40 \mathrm{~m}$ et $43 \mathrm{~m}$. Le test Kruskal-Wallis montre une variation significative $(\mathrm{p}<0,0001)$ de la densité moyenne en fonction de l'altitude moyenne des inselbergs des différents secteurs phytogéographiques. En effet, la végétation est plus dense sur les inselbergs des secteurs nord-sahélien et sud-souda- nien qui ont les plus fortes hauteurs moyennes (respectivement $57 \mathrm{~m}$ et $62 \mathrm{~m}$ ).

Les variations de la surface terrière moyenne sont en accord avec la proportion et la densité moyenne des individus adultes et juvéniles dans les différents secteurs. Plus le nombre et la densité moyenne des juvéniles sont élevés plus la surface terrière est faible. La surface terrière, la proportion et la densité moyenne de juvéniles obtenues dans le secteur nord-soudanien illustrent cet état de fait. L'état des peuplements ligneux dans les secteurs sud-sahélien et nord-soudanien pourrait s'expliqué par la forte anthropisation dans ces secteurs. Dans certaines localités de ces deux secteurs nous avons pu noter la pratique de culture de mil et de riz sur les inselbergs. Ceci s'expliquerait par l'explosion démographique qui amènerait les populations et le bétail à s'orienter vers les inselbergs qui voit leur végétation se dégradée au fur et à mesure que la pression s'augmente.

Les données ont été collectées à la même période (mi-août; début septembre) dans les différents secteurs phytogéographiques. Nous pouvons alors dire que la bonne proportion des juvéniles dans les secteurs nord-sahélien, sud-sahélien et sud-soudanien assurerait une survie aux peuplements ligneux des inselbergs de ces secteurs. 


\subsection{Facteurs influençant la végétation ligneuse des insel- bergs}

La végétation ligneuse des inselbergs est influencée par les facteurs climatique, topographique et anthropique. L'influence du climat sur la végétation ligneuse des inselbergs s'illustre d'une part par l'augmentation du nombre moyen d'espèces par relevé (Tableau 1) et d'autre part par la croissance du diamètre moyen le long du gradient climatique nord-sud (Tableau 5). Cette augmentation du nombre d'espèces et du diamètre moyen est due à la variation des conditions climatiques, particulièrement la pluviométrie qui devient de plus en plus abondante du nord au sud du Burkina Faso. La dissimilitude de la richesse floristique entre le secteur nord-sahélien et les secteurs nord-soudanien et sud-soudanien étaie l'influence du climat sur la végétation ligneuse des inselbergs. En effet, les indices de similitude de Sorensen entre le secteur nord-sahélien et les secteurs nord-soudanien et sud-soudanien sont respectivement $35 \%$ et $19 \%$. L'augmentation de la proportion des Rubiaceae du Secteur nord-sahélien (3\%) vers le secteur Sud-soudanien (11\%) met également en évidence l'influence du climat sur la végétation ligneuse des inselbergs. Nos résultats sont en accord avec ceux de SAVAdogo (2012). Selon Aubreville (1950) les Rubiaceae arborescentes et arbustives sont caractéristiques des forêts denses humides. Nos résultats sont en conformité avec d'autres études antérieures (Thiombiano 2005; Thiombiano et al. 2006; Bognounou et al. 2009; SAMBARÉ et al. 2010; Schmidt et al. 2013) qui ont souligné la prédominance du climat, précisément la pluviométrie dans la répartition des taxons ligneux du secteur nord-sahélien au secteur sud-soudanien.

La composition floristique d'une part et d'autre part la densité moyenne et la surface terrière moyenne varient en fonction des strates (sommets et versants). Ces variations attestent l'influence de la topographie sur la végétation des inselbergs. La variation significative du taux d'exploitation $(\mathrm{p}<0,0001)$ entre les sommets et les versants indique également l'influence de la topographie sur la végétation ligneuse.

Les traces d'exploitation humaine relevées sur les ligneux et la pratique de l'agriculture sur certains inselbergs témoignent de l'influence du facteur anthropique sur la végétation ligneuse des inselbergs. En effet, sur un total de 11060 individus recensés, $8 \%$ de ces individus portent les traces d'exploitation humaine. En outre, sur un total de 16 inselbergs étudiés l'agriculture est pratiquée sur 2 d'entre eux, soit $13 \%$ de pratique d'agriculture. Les traces de feux de brousse ont été notées sur les inselbergs des secteurs nordsoudanien et sud-soudanien.

\section{CONCLUSION}

Avec 143 espèces ligneuses inventoriées, l'étude de la végétation ligneuse des inselbergs contribue à la connaissance de la répartition des taxons au Burkina Faso. Les inselbergs regorgent à eux seuls $65 \%$ des espèces ligneuses et $7 \%$ de la flore totale du Burkina Faso. Les inselbergs du secteur sud-soudanien renferment à eux seuls $80 \%$ des espèces ligneuses recensées. Bien qu'ayant la faible richesse floristique, les inselbergs du secteur nord-sahélien apportent leur contribution à la phytodiversité par des taxons qui leur sont propres. Douze espèces menacées du Burkina Faso font parties des espèces dominantes des inselbergs et traduisent de ce fait le caractère refuge des inselbergs. Il ressort de cette étude que les facteurs influençant la végétation ligneuse des inselbergs sont le climat, la topographie et la pression anthropisation. Mais de tous ces facteurs le climat reste le principal facteur qui influe sur la répartition des taxons ligneux. Nos hypothèses émises dès le départ ont été confirmées. Il existe différents types d'habitats et de communautés végétales sur les inselbergs qu'il serait important de les étudier suivant le gradient climatique. Pour une meilleure connaissance et la conservation des espèces végétales des inselbergs nous reformulons les recommandations suivantes à l'endroit du Ministère de l'Environnement et les partenaires financiers:

- Encourager et soutenir la recherche sur les inselbergs,

- Classer les inselbergs parmi les aires protégées,

- Sensibiliser les populations locales sur la préservation et la protection des ressources végétales des inselbergs.

\section{REMERCIEMENTS}

Nous adressons nos sincères remerciements au Dr. Mahamadi DiandaI pour la contribution au financement de nos travaux de terrain. Nous remercions également Monsieur Abel Kabeda d'avoir financé une partie de nos travaux de terrain et pour la réalisation de la carte. Nous remercions le Professeur Stefan Pormebski pour son assistance technique lors de la collecte des données. Nos sincères remerciements vont en direction des autorités locales et groupes socioculturels des villages environnants des inselbergs des quatre secteurs phytogéographiques.

\section{REFERENCES}

Akoegninou A, van der Burg WJ \& VAN Der MAesen LJG (2006): Flore Analytique du Bénin. Backhuys Publishers, Wageningen, $1034 \mathrm{p}$.

Arbonier M (2009): Arbres, arbustes et lianes des zones sèches d'Afrique de l'Ouest. Quæ, MNHN., 573 p.

Aubreville A (1950): Flore forestière soudano-guinéenne : AOF ; Cameroun: AEF. Société d'édition géographique, Paris, $523 \mathrm{p}$.

Barthlott W, Gröger A. \& Porembski S (1993): Some remarks on the vegetation of tropical inselbergs: diversity and ecological differentiation. Biogeogr 69 (3): 105-124.

Berhaut J (1967): Flore du Sénégal, 2ème édition, Clairafrique, $485 \mathrm{p}$.

Berhaut J (1971): Flore illustrée du Sénégal. Dicotylédones, Tome I : Acanthacées à Avicenniacées. Gouvernement du Sénégal, Ministère du Développement Rural, Dakar, 626 p.

Berhaut J (1974): Flore illustrée du Sénégal. Dicotylédones, Tome II : Balanophoracées à Composées. Gouvernement du Sénégal, Ministère du Développement Rural, Dakar, 695 p.

BERHAUt J (1975): Flore illustrée du Sénégal. Dicotylédones, Tome III : Connaracées à Euphorbiacées. Gouvernement du Sénégal, Minitère du Développement Rural, Dakar, $634 \mathrm{p}$. 
Berhaut J (1976): Flore illustrée du Sénégal. Dicotylédones, Tome V : Légumineuses et Papillionacées. Gouvernement du Sénégal, Ministère du Développement Rural, Dakar, 658 p.

Berhaut J (1988): Flore illustrée du Sénégal. Monocotylédones et Ptéridophytes, Tome IX, Monocotylédones : Agavacées à Orchidacées. Gouvernement du Sénégal, Ministère du Développement Rural, Dakar, 523 p

Bognounou F (2009): Restauration écologique et gradient latitudinal: utilisation, diversité et régénération de cinq espèces de Combretaceae au Burkina Faso. Doctorat de l'Université de Ouagadougou. Unité de Recherche et de Formation en Sciences de la vie et de la Terre, 179 p.

Bognounou F, Thiombiano A, Savadogo P, Boussim J.I, ODEN PC \& GUINKo S (2009): Woody vegetation structure and composition at four sites along latitudinal gradient in Western Burkina Faso. Bois Forêts Tropiques, 300 (2): 2944.

Fontès J \& GuInKo S (1995): Carte de la végétation et de l'occupation des sols du Burkina Faso. Notice explicative, Ministère de la coopération français, projet Campus, Toulouse, $68 \mathrm{p}$.

GANABA S (1994): Rôle des systèmes racinaires dans la dynamique du peuplement ligneux de la région de la d'Oursi (Burkina Faso) entre 1980 et 1982. Thèse de 3ème cycle. Université de Ouagadougou, Faculté des Sciences et Techniques, $195 \mathrm{p}$.

GANABA S (2008): Caractérisation, utilisation, tests de restauration et gestion de la végétation ligneuse au Sahel, Burkina Faso. Thèse de Doctorat d'Etat ès Sciences Naturelle. Université Cheikh Anta-Diop n ${ }^{\circ} 117$. Faculté des Sciences et Techniques, $287 \mathrm{p}$.

HANNAH R \& KRYSTYNA S (2008): Biodiversité, changement climatique et pauvreté. Rapport de l'IIED (International Institute for Environment and Development), 8 p.

Hutchinson J, Dalziel J.M (1958): Flora of West Tropical Africa. 2è ed.. Volume I, Part 2. Crown Agents for Oversea Governments and administrations, Milbank, London, 828 p.

Hutchinson J, Dalziel J.M (1963): Flora of West Tropical Africa. 2è ed.. Volume II, Part 2. Crown Agents for Oversea Governments and administrations, Milbank, London, 544 p.

Hutchinson J, DAlziel J.M, (1954): Flora of West Tropical Africa. 2è ed.. Volume I, Part 1. Crown Agents for Oversea Governments and administrations, Milbank, London, 295 p.

Inoussa TM, Ismaila TI, Médard CG \& BRICE S (2013): Structure et composition floristiques des forets denses sèches des Monts kouffé. J Appl Biosci 64: 4787 - 4796.

Kouassi RH, TiÉBrÉ M-S \& N'Guessan KE (2009): Aperçu de la végétation des Inselbergs Brafouéby et Mafa-Mafou (Sud-Est de la Côte d'Ivoire). European J Sci Research 28

KüPPERS K, \& WiTtig R (1995): Überblick über die vegetation der chaine de Gobnangou (Burkina Faso, Westafrica). Verhandlungen Ges Ökol 24: 27-30

LARRERE C \& LARRERE R (1997): Du bon usage de la nature. Pour une philosophie de l'environnement. Aubier, Paris, 355 p.

Mbayngone E, Thiombiano A, Hahn-Hadjali K \& GuinKO S (2008): Caractéristiques écologiques de la végétation ligneuse du sud-est du Burkina Faso (Afrique de l'Ouest) : le cas de la réserve de Pama. Candollea 63: 17-33.
Ministère DE L'ENVIRONNEMENT ET DU DéVELOPPEMENT Durable (2011): Programme d'Investissement Forestier (PIF - Burkina Faso). Vol 2. 70 p.

MüLLER J (2008): Herbaceous and non-inundated vegetation of Sahelian inselbergs in Burkina Faso. Candollea 63: 57-79.

OuÉDRAOGo A (2006): Diversité et dynamique de la végétation ligneuse de la partie orientale du Burkina Faso. Thèse de doctorat de l'Université de Ouagadougou. UFR/SVT, $195 \mathrm{p}$.

OuÉDRAOGo A \& Thiombiano A (2012): Regeneration pattern of four threatened tree species in Sudanian savannas of Burkina Faso. Agroforestry Systems 861: 35-48.

OuÉDraogo O (2009): Phytosociologie, dynamique et productivité de la végétation du parc national d'Arly (Sud-Est du Burkina Faso). Thèse de doctorat de l'Université de Ouagadougou. UFR/SVT, $188 \mathrm{p}$.

Ouédraogo O, Thiombiano A, Hahn-Hadjali K \& GuinKO S (2008): Diversité et structure des groupements ligneux du parc national d'Arly (Est du Burkina Faso). Flora Veg Sudano-Sambesica 11: 5-16.

Ouoba P (2006): Flore et végétation de la forêt classée de Niangoloko, Sud-Ouest du Burkina Faso. Thèse de doctorat, Université de Ouagadougou, 144 p.

Parmentier I, Oumorou M, Porembski S, Lejoly J, DeCOCQ G (2006): Ecology, distribution, and classification of xeric monocotyledonous mats on inselbergs in West Africa and Atlantic central Africa. Phytocoenol 36: 547-564.

POREMBSKI S (2007): Effects of anthropogenic disturbance on the vegetation of granitic and gneissic rock outcrops ('inselberg') in West Africa. Nova Hedwigia, Beiheft 131: 237246

Sambaré O, Ouédraggo O, Wittig R et Thiombiano A (2010): Diversité et écologie des groupements ligneux des formations ripicoles du Burkina Faso (Afrique de l'Ouest). Internat J Biol Chem Sci 4: 1782-1800.

Sambaré O, Bognounou F, Wittig R, Thiombiano A (2011): Woody species composition, diversity and structure of riparian forests of four watercourses types in Burkina Faso. J Forestry Research 22: 145-158.

SAVADOGo S (2013): Les bois sacrés du Burkina Faso: diversité, structure, dimension spirutuelle et mode de gestion de leurs ressources naturelles. Thèse de doctorat de l'Université de Ouagadougou. UFR/SVT, 280 p.

Savadogo S, OuÉdraogo A, Thiombiano A (2010): Perceptions, mode de gestion et végétation des bois sacrés au nord du Burkina Faso. Flora Veg Sudano-Sambesica 13: 1021.

Schmidt M, Traoré S, OuÉDraogo A, Mbayngone E, Oú́draogo O, Zizka A, Kirchmair I, Kaboré E, Tindano E, Thiombiano A, Hahn K \& Zizka G (2013): Geographical patterns of woody plants' functionaltraits in Burkina Faso. Candollea 68: 197-207.

Sinsin B (2001): Formes de vie et diversité spécifique des associations de forêts claires du nord du Bénin. XVIth AETFAT Congress Syst Geogr 71: 873-888.

Sinsin B \& KampManN D (eds) (2010): Atlas de la biodiversité de l'Afrique de l'ouest, Tome I : Bénin. Cotonou et frankfurt/Main. BIOTA, $676 \mathrm{p}$.

Thiombiano A (1996): Contribution à l'étude des Combretaceae dans les formations végétales de la région Est du 
Burkina Faso. Thèse de 3ème cycle. Université de Ouagadougou. FAST., 200 p. +6 annexes.

Thiombiano A (2005): Les Combretaceae du Burkina Faso: taxonomie, écologie, dynamique et régénération des espèces. Thèse de Doctorat d'Etat, Université de Ouagadougou, $290 \mathrm{p}$.

Thiombiano A \& Kampmann D (eds) (2010): Atlas de la biodiversité de l'Afrique de l'ouest, Tome II : Burkina Faso. Ouagadougou et Frankfurt/Main, 592 p.

SINSIN B (2001): Formes de vie et diversité spécifique des associations de forêts claires du nord du Bénin. XVIth AETFAT Congress Syst Geogr 71: 873-888.

Thiombiano A, Schmidt M, Dressler S, OuÉDraogo A, HAHN K \& ZizKa G (2012): Catalogue des plantes vasculaires du Burkina Faso. Boissiera 65, 391 pp.

Thiombiano A, Schmidt M, Kreft H, \& Guinko S (2006): Influence du gradient climatique sur la distribution des espèces Combretaceae au Burkina Faso (Afrique de l'Ouest). Candollea 61: 189-213 p.

Tindano E, Ganaba S, Thiombiano A (2011): Rocky woody vegetation diversity and structure in the Oursi dam area Northern Burkina Faso. ISESCO Sci Technol 7 (12): 15-28.
Traoré K et Mangara A (2009): Etude Phyto-Ecologique des Adventices dans les Agro-Ecosystèmes Elaeicoles de la Mé et de Dabou. European J Sci Research 31 : 519-533

Sinsin B (2001): Formes de vie et diversité spécifique des associations de forêts claires du nord du Bénin. XVIth AETFAT Congress Syst Geogr 71: 873-888.

Traoré L, OuÉDraogo I, OuÉDraogo A \& Thiombiano A, (2011): Perceptions, usages et vulnérabilité des ressources végétales ligneuses dans le Sud-Ouest du Burkina Faso. Internat J Biol Chem Sci 5: 258-278.

Traoré L, Sop TK, Dayamba SD, Traoré S, Hahn K, \& Thiombiano A. (2013) : Do protected areas really work to conserve species? A case study of three vulnerable woody species in the Sudanian zone of Burkina Faso. Environment, Development Sustainability 15: 663-686.

White M (1986): La végétation de l'Afrique. Mémoire accompagnant la carte de végétation de l'Afrique UNESCO/ AETFAT/UNSO. ORSTOM/UNESCO, 389 P.

Wittig R, Hahn-Hadjali K \& Thiombiano A (2000): Les particularités de la végétation et de la flore de la chaîne du Gobnangou dans le sud-est du Burkina Faso. Etudes Flor. Vég Burkina Faso 5: 49-64.

E-mail adresses: elisetindano82@yahoo.fr, ganabasouley@ gmail.com, adjima_thiombiano@yahoo.fr 